\title{
Planetary Candidates Observed by Kepler III: Analysis of the First 16 Months of Data
}

\section{Citation}

Batalha, Natalie M., Jason F. Rowe, Stephen T. Bryson, Thomas Barclay, Christopher J. Burke, Douglas A. Caldwell, Jessie L. Christiansen, et al. 2013. Planetary Candidates Observed by Kepler III: Analysis of the First 16 Months of Data. The Astrophysical Journal Supplement Series 204, no. 2: 24. doi:10.1088/0067-0049/204/2/24.

\section{Published Version}

doi:10.1088/0067-0049/204/2/24

\section{Permanent link}

http://nrs.harvard.edu/urn-3:HUL.InstRepos:29990201

\section{Terms of Use}

This article was downloaded from Harvard University's DASH repository, and is made available under the terms and conditions applicable to Other Posted Material, as set forth at http:// nrs.harvard.edu/urn-3:HUL.InstRepos:dash.current.terms-of-use\#LAA

\section{Share Your Story}

The Harvard community has made this article openly available.

Please share how this access benefits you. Submit a story.

\section{Accessibility}




\title{
PLANETARY CANDIDATES OBSERVED BY KEPLER. III. ANALYSIS OF THE FIRST 16 MONTHS OF DATA
}

\author{
Natalie M. Batalha ${ }^{1,2}$, Jason F. Rowe ${ }^{3}$, Stephen T. Bryson $^{2}$, Thomas Barclay ${ }^{4}$, Christopher J. Burke ${ }^{3}$, \\ Douglas A. Caldwell ${ }^{3}$, Jessie L. Christiansen ${ }^{2}$, Fergal Mullally $^{3}$, Susan E. Thompson ${ }^{3}$, Timothy M. Brown ${ }^{5}$, \\ Andrea K. Dupree ${ }^{6}$, Daniel C. Fabrycky ${ }^{7}$, Eric B. Ford ${ }^{8}$, Jonathan J. Fortney ${ }^{7}$, Ronald L. Gilliland ${ }^{9}$,
} Howard IsaACson $^{10}$, David W. Latham ${ }^{6}$, Geoffrey W. Marcy ${ }^{10}$, SAmuel N. Quinn ${ }^{6,11}$, Darin Ragozzine ${ }^{6}$, Avi ShPorer ${ }^{5}$, William J. Borucki ${ }^{2}$, David R. Ciardi ${ }^{12}$, Thomas N. Gautier III ${ }^{13}$, Michael R. HaAs ${ }^{2}$, Jon M. Jenkins ${ }^{3}$, David G. Koch ${ }^{2}$, JaCk J. Lissauer $^{2}$, William Rapin ${ }^{2}$, Gibor S. Basri ${ }^{10}$, Alan P. Boss ${ }^{14}$, Lars A. Buchhave ${ }^{15,16}$, Joshua A. Carter ${ }^{6}$, David Charbonneau ${ }^{6}$, Joergen Christensen-Dalsgaard ${ }^{17}$, Bruce D. Clarke ${ }^{13}$, William D. Cochran ${ }^{18}$, Brice-Olivier Demory ${ }^{19}$, Jean-Michel Desert ${ }^{6}$, Edna Devore ${ }^{20}$, Laurance R. Doyle ${ }^{20}$, Gilbert A. Esquerdo ${ }^{6}$, Mark EveretT $^{21}$, Francois Fressin ${ }^{6}$, John C. Geary ${ }^{6}$, Forrest R. Girouard ${ }^{22}$, Alan Gould ${ }^{23}$, Jennifer R. Hall ${ }^{22}$, Matthew J. Holman ${ }^{6}$, Andrew W. Howard ${ }^{10}$, Steve B. Howell ${ }^{2}$, Khadeejah A. Ibrahim ${ }^{22}$, Karen Kinemuchi ${ }^{4}$, Hans KJeldsen ${ }^{17}$, Todd C. Klaus ${ }^{22}$, Jie Li $^{3}$, Philip W. Lucas ${ }^{24}$, Søren Meibom ${ }^{6}$, Robert L. Morris ${ }^{22}$, Andrej PrŠa ${ }^{25}$, Elisa Quintana ${ }^{3}$, Dwight T. Sanderfer ${ }^{22}$, Dimitar Sasselov ${ }^{6}$, Shawn E. Seader ${ }^{3}$, JefFrey C. Smith ${ }^{3}$, Jason H. StefFen ${ }^{26}$, Martin Still ${ }^{4}$, Martin C. Stumpe ${ }^{3}$, Jill C. Tarter ${ }^{20}$, Peter Tenenbaum $^{3}$, Guillermo Torres $^{6}$, Joseph D. Twicken ${ }^{3}$, Kamal UdDin ${ }^{22}$, JefFrey Van Cleve ${ }^{3}$, LuCianne Walkowicz ${ }^{27}$, and William F. Welsh ${ }^{28}$

${ }^{1}$ Department of Physics and Astronomy, San Jose State University, San Jose, CA 95192, USA; Natalie.Batalha@ nasa.gov ${ }^{2}$ NASA Ames Research Center, Moffett Field, CA 94035, USA ${ }^{3}$ SETI Institute/NASA Ames Research Center, Moffett Field, CA 94035, USA

${ }^{4}$ Bay Area Environmental Research Institute/NASA Ames Research Center, Moffett Field, CA 94035, USA

${ }^{5}$ Las Cumbres Observatory Global Telescope Network, Goleta, CA 93117, USA

${ }^{6}$ Harvard-Smithsonian Center for Astrophysics, 60 Garden Street, Cambridge, MA 02138, USA

${ }^{7}$ Department of Astronomy and Astrophysics, University of California, Santa Cruz, CA 95060, USA

${ }^{8}$ Department of Astronomy, University of Florida, Gainesville, FL 32611, USA

${ }^{9}$ Center for Exoplanets and Habitable Worlds, The Pennsylvania State University, University Park, PA 16802, USA

${ }^{10}$ Department of Astronomy, University of California Berkeley, Berkeley, CA 94720, USA

${ }^{11}$ Department of Physics and Astronomy, Georgia State University, PO Box 4106, Atlanta, GA 30302, USA

${ }_{12}$ NASA Exoplanet Science Institute/Caltech, Pasadena, CA 91125, USA

${ }^{13}$ Jet Propulsion Laboratory/California Institute of Technology, Pasadena, CA 91109, USA

${ }^{14}$ Carnegie Institution of Washington, Washington, DC 20015-1305, USA

${ }^{15}$ Niels Bohr Institute, University of Copenhagen, DK-2100 Copenhagen, Denmark

${ }^{16}$ Centre for Star and Planet Formation, Natural History Museum of Denmark, University of Copenhagen, DK-1350 Copenhagen, Denmark

${ }^{17}$ Department of Physics and Astronomy, Aarhus University, DK-8000 Aarhus C, Denmark

${ }^{18}$ McDonald Observatory, The University of Texas, Austin, TX 78712, USA

${ }^{19}$ Department of Earth, Atmospheric and Planetary Sciences, Massachusetts Institute of Technology, 77 Massachusetts Avenue, Cambridge, MA 02139, USA

${ }^{20}$ SETI Institute, Mountain View, CA 94043, USA

${ }^{21}$ National Optical Astronomy Observatory, Tucson, AZ 85719, USA

22 Orbital Sciences Corporation/NASA Ames Research Center, Moffett Field, CA 94035, USA

${ }^{23}$ Lawrence Hall of Science, Berkeley, CA 94720, USA

${ }^{24}$ Centre for Astrophysics, University of Hertfordshire, College Lane, Hatfield AL10 9AB, UK

${ }^{25}$ Department of Astronomy and Astrophysics, Villanova University, Villanova, PA 19085, USA

${ }^{26}$ Fermilab Center for Particle Astrophysics, Batavia, IL 60510, USA

${ }^{27}$ Department of Astrophysical Sciences, Princeton University, Princeton, NJ 08544, USA

${ }^{28}$ Department of Astronomy, San Diego State University, San Diego, CA 92182, USA

Received 2012 February 27; accepted 2012 November 14; published 2013 February 5

\begin{abstract}
New transiting planet candidates are identified in 16 months (2009 May-2010 September) of data from the Kepler spacecraft. Nearly 5000 periodic transit-like signals are vetted against astrophysical and instrumental false positives yielding 1108 viable new planet candidates, bringing the total count up to over 2300. Improved vetting metrics are employed, contributing to higher catalog reliability. Most notable is the noise-weighted robust averaging of multiquarter photo-center offsets derived from difference image analysis that identifies likely background eclipsing binaries. Twenty-two months of photometry are used for the purpose of characterizing each of the candidates. Ephemerides (transit epoch, $T_{0}$, and orbital period, $P$ ) are tabulated as well as the products of light curve modeling: reduced radius $\left(R_{\mathrm{P}} / R_{\star}\right)$, reduced semimajor axis $\left(d / R_{\star}\right)$, and impact parameter $(b)$. The largest fractional increases are seen for the smallest planet candidates (201\% for candidates smaller than $2 R_{\oplus}$ compared to $53 \%$ for candidates larger than $2 R_{\oplus}$ ) and those at longer orbital periods (124\% for candidates outside of 50 day orbits versus $86 \%$ for candidates inside of 50 day orbits). The gains are larger than expected from increasing the observing window from 13 months (Quarters 1-5) to 16 months (Quarters 1-6) even in regions of parameter space where one would have expected the previous catalogs to be complete. Analyses of planet frequencies based on previous catalogs will be affected by such incompleteness. The fraction of all planet candidate host stars with multiple candidates has grown from $17 \%$ to $20 \%$, and the paucity of short-period giant planets in multiple systems is still evident. The progression
\end{abstract}


toward smaller planets at longer orbital periods with each new catalog release suggests that Earth-size planets in the habitable zone are forthcoming if, indeed, such planets are abundant.

Key words: catalogs - eclipses - planetary systems - space vehicles - techniques: photometric

Online-only material: color figures, machine-readable tables

\section{INTRODUCTION}

Since initiating science operations in 2009 May, Kepler has produced two catalogs of transiting planet candidates. The first, released in 2010 June, contains 312 candidates identified in the first 43 days of Kepler data (Borucki et al. 2011a) and is hereafter referred to as B10. The second, released in 2011 February, is a cumulative catalog containing 1235 candidates identified in the first 13 months (Quarters 1-5) (29 $^{29}$ of data (Borucki et al. 2011b). This cumulative catalog is hereafter referred to as B11. Over 60 candidates from the B11 catalog have been confirmed, including many of Kepler's milestone discoveries: the mission's first rocky planet, Kepler-10b (Batalha et al. 2011); the sixtransiting-planet system, Kepler-11 (Lissauer et al. 2011a); the first circumbinary planet, Kepler-16ABb (Doyle et al. 2011); the $2.38 R_{\oplus}$ planet in the habitable zone (HZ), Kepler-22b (Borucki et al. 2012); and the mission's first Earth-size planets, Kepler-20 e \& f (Fressin et al. 2012).

In this contribution, we present new planet candidates identified from the analysis of 16 months of data (Quarters 1-6). The analysis was motivated by the availability of the SOC 7.0 pipeline in the summer of 2011. A new catalog would not necessarily have been warranted by the addition of only one quarter of data. However, the new multi-quarter functionality of Kepler's pipeline transit search module (Transiting Planet Search, TPS) yielded candidates that were missed in previous catalogs that used ad hoc tools for multi-quarter transit searches to detect long-period planet candidates.

We describe the results of this effort-the data (Section 2), the procedures that sort transit-like signals coming out of the pipeline into viable planet candidates (Section 3), and the subsequent vetting criteria that lead to increased catalog reliability (Section 4). We describe the characterization of the planet candidates (Section 5) that begins with transit light curve modeling (Section 5.1) and ultimately requires detailed knowledge of the stellar properties. An effort was made to improve upon the stellar properties from the Kepler Input Catalog (KIC; Brown et al. 2011) by utilizing theoretical evolutionary tracks as described in Section 5.2. We examine the distributions of the resulting planet properties (Section 6) and take a collective look at the progress to date as we work toward the identification of Earth-size planets in the HZ. We compare the observed gains to those predicted by way of adding three months of data (Section 7.1). The new multiple transiting planet systems are briefly described, as are the candidates in the HZ. Finally, in the Appendix, we provide a cumulative table of planet candidates containing the characteristics of the new candidates as well as updated characteristics of the candidates in the B11 catalog computed using the same data used herein.

\footnotetext{
29 Quarters are defined by a requirement to roll the spacecraft $90^{\circ}$ about its axis to keep the solar arrays illuminated and the focal-plane radiator pointed away from the Sun. All but the first quarter are approximately 93 days in duration. In Quarter 1, the spacecraft operated in science mode for 33 days.
}

\section{OBSERVATIONS}

The data employed for transit identification were acquired between 2009 May 13 00:15 UTC and 2010 Sep 22 19:03 UTC (Q1-Q6). Over 190,000 stars were observed at some time during this period. Of these, only 127,816 were observed every single quarter. Therefore, it should not be assumed that every star tabulated herein was observed continuously for the six quarter period. While this is a reasonable assumption for the 2011 February catalog (906, or $91 \%$ of the 997 stars identified as planet hosts were observed all five quarters), it is not for the population of new candidates presented here, where only 704 $(76 \%)$ of the 926 unique stars identified as planet hosts were observed all six quarters. The last column of Table 3 presents a string of six integers, each indicating if the target was (one) or was not (zero) observed during the quarter in question (ordered one through six, from left to right). The 4th integer (corresponding to Quarter 4) can also assume a value of 2, indicating targets located on CCD Module 3 during Quarter 4. Module 3 failed at 17:52 UTC on 2010 January 9 and never recovered. Targets located on that module were observed for a shorter time period (see below). The start and stop times for each quarter are listed in Table 1 . The loss of Module 3 implies that approximately $19 \%$ of Kepler's targets will be observed three out of four quarters each year.

The data employed were taken at long-cadence (LC) whereby 270 readouts of slightly more than 6.5 second duration (6.01982 s integration and $0.51895 \mathrm{~s}$ read time) are co-added to 29.4 minute intervals. Quarters 1-6 yield flux time series with 1,639, 4,354, 4,370, 4,397, 4,633, and 4,397 cadences (see also Table 1) corresponding to $33.5,88.9,89.3,90.3,94.7$, and 89.8 days of photometry, respectively. The exception to this is the number of cadences in Quarter 4 for targets falling on CCD Module 3 (channels 5, 6, 7, and 8). Such targets were observed for 1022 cadences instead of 4397. Besides the interruption for some targets due to the Module 3 failure, each quarterly time series contains gaps, some larger than others, due to a variety of occurrences including monthly breaks for data downlink, occasional safe mode events, manually excluded cadences, loss of fine point, and attitude tweaks. All missing cadences are tabulated in the Anomaly Summary Table in Section 5 of the Data Release Notes (DRN) archived at MAST. ${ }^{30}$ Also included in the DRN are the start and stop time of each quarter. This information, together with the transit ephemerides presented in Table 4 is sufficient for reconstructing the number of observed transits in time series of any length.

Pixel data are converted to instrumental fluxes via Kepler pipeline software modules that calibrate pixel data (Quintana et al. 2010), perform aperture photometry (Twicken et al. 2010a), and correct for systematic errors (Twicken et al. 2010b). The pipeline software is documented in the Kepler Data Processing Handbook (KSCI-19081) at MAST. As described in Section 2 of that document, each data set is associated with a software release

\footnotetext{
30 Multi-Mission Archive at Space Telescope Science Institute; http://archive.stsci.edu/kepler
} 
Table 1

Data Collection Times

\begin{tabular}{|c|c|c|c|c|c|}
\hline \multirow[t]{2}{*}{ Quarter } & \multicolumn{2}{|c|}{ First Cadence Mid-time } & \multicolumn{2}{|c|}{ Last Cadence Mid-time } & \multirow[t]{2}{*}{$N_{\text {cadence }}$} \\
\hline & (MJD) & (UTC) & (MJD) & (UTC) & \\
\hline 1 & 54964.011 & 2009 May 13 00:15 & 54997.481 & 2009 Jun 15 11:32 & 1639 \\
\hline 2 & 55002.0175 & 2009 Jun 20 00:25 & 55090.9649 & 2009 Sep 16 23:09 & 4354 \\
\hline 3 & 55092.7222 & 2009 Sep 18 17:19 & 55181.9966 & 2009 Dec 16 23:55 & 4370 \\
\hline 4 & 55184.8778 & 2009 Dec 19 21:04 & 55274.7038 & 2010 Mar 19 16:53 & 4397 \\
\hline 5 & 55275.9912 & 2010 Mar 20 23:47 & 55370.6600 & 2010 Jun 23 15:50 & 4633 \\
\hline 7 & 55462.6725 & 2010 Sep 23 16:08 & 55552.0491 & 2010 Dec 22 01:10 & 4375 \\
\hline 8 & 55567.8647 & 2011 Jan 06 20:45 & 55634.8460 & 2011 Mar 14 20:18 & 3279 \\
\hline
\end{tabular}

Notes. Quarters 1-8 are only employed in the light curve modeling used to derive the planet candidate properties. Transits are identified on a pipeline run using Quarters 1-6 only. CCD Module 3 failed in Quarter 4 on 2010 January 9 at 17:52 UTC. Targets located on Module 3 during this quarter were only observed for 1022 cadences.

Table 2

KOIs Noted as V-shaped

\begin{tabular}{|c|c|c|c|c|c|c|c|c|c|c|c|}
\hline KOI & $\begin{array}{c}P \\
\text { (days) }\end{array}$ & $\frac{R_{\mathrm{P}}}{R_{\star}}$ & $1-b-\frac{R_{\mathrm{P}}}{R_{\star}}$ & KOI & $\begin{array}{c}P \\
\text { (days) }\end{array}$ & $\frac{R_{\mathrm{P}}}{R_{\star}}$ & $1-b-\frac{R_{\mathrm{P}}}{R_{\star}}$ & KOI & $\begin{array}{c}P \\
\text { (days) }\end{array}$ & $\frac{R_{\mathrm{P}}}{R_{\star}}$ & $1-b-\frac{R_{\mathrm{P}}}{R_{\star}}$ \\
\hline 51.01 & 10.43 & 0.43 & -0.632 & 886.03 & 21.00 & 0.03 & -0.003 & 1793.01 & 3.26 & 0.30 & -0.541 \\
\hline 113.01 & -387 & 0.57 & -0.955 & 976.01 & 52.57 & 0.50 & -0.791 & 1798.01 & 12.96 & 0.08 & -0.050 \\
\hline 138.01 & 48.94 & 0.12 & -0.108 & 1020.01 & 54.36 & 0.37 & -0.623 & 1799.01 & 1.73 & 0.47 & -0.821 \\
\hline 151.01 & 13.45 & 0.05 & -0.027 & 1032.01 & -650 & 0.09 & -0.060 & 1829.01 & 22.84 & 0.33 & -0.601 \\
\hline 225.01 & 0.84 & 0.43 & -0.810 & 1095.01 & 51.60 & 0.09 & -0.020 & 1845.02 & 5.06 & 0.29 & -0.540 \\
\hline 256.01 & 1.38 & 0.44 & -0.678 & 1096.01 & -414 & 0.11 & 99.886 & 1872.01 & 30.52 & 0.08 & -0.074 \\
\hline 371.01 & 498.39 & 0.30 & -0.556 & 1118.01 & 7.37 & 0.02 & -0.011 & 1906.01 & 8.71 & 0.18 & -0.323 \\
\hline 403.01 & 21.06 & 0.40 & -0.767 & 1192.01 & -201292 & 0.32 & -0.539 & 1935.01 & 15.44 & 0.14 & -0.204 \\
\hline 410.01 & 7.22 & 0.36 & -0.656 & 1193.01 & 119.06 & 0.09 & -0.037 & 1944.01 & 12.18 & 0.03 & -0.005 \\
\hline 417.01 & 19.19 & 0.12 & -0.106 & 1209.01 & 272.07 & 0.08 & -0.009 & 1968.01 & 10.09 & 0.03 & -0.006 \\
\hline 419.01 & 20.13 & 0.33 & -0.549 & 1226.01 & 137.76 & 0.40 & -0.351 & 2042.01 & 63.07 & 0.03 & -0.000 \\
\hline 466.01 & 9.39 & 0.08 & -0.049 & 1227.01 & 2.16 & 0.31 & -0.417 & 2128.01 & 24.26 & 0.24 & -0.435 \\
\hline 473.01 & 12.71 & 0.04 & -0.001 & 1242.01 & 99.64 & 0.43 & -0.797 & 2156.01 & 2.85 & 0.06 & -0.034 \\
\hline 601.02 & 11.68 & 0.23 & -0.421 & 1359.02 & 104.82 & 0.07 & -0.014 & 2189.01 & 33.36 & 0.47 & -0.901 \\
\hline 609.01 & 4.40 & 0.12 & -0.123 & 1385.01 & 18.61 & 0.60 & -0.935 & 2204.01 & 10.86 & 0.02 & -0.005 \\
\hline 611.01 & 3.25 & 0.10 & -0.093 & 1387.01 & 23.80 & 0.44 & -0.555 & 2259.01 & 12.19 & 0.23 & -0.441 \\
\hline 614.01 & 12.87 & 0.08 & -0.039 & 1409.01 & 16.56 & 0.03 & -0.003 & 2299.01 & 16.49 & 0.10 & -0.162 \\
\hline 617.01 & 37.87 & 0.41 & -0.720 & 1426.03 & 150.03 & 0.15 & -0.196 & 2363.01 & 3.14 & 0.02 & -0.003 \\
\hline 620.03 & 85.31 & 0.06 & -0.029 & 1502.01 & 1.88 & 0.03 & -0.001 & 2370.01 & 78.73 & 0.03 & -0.019 \\
\hline 625.01 & 38.14 & 0.18 & -0.324 & 1540.01 & 1.21 & 0.38 & -0.430 & 2380.01 & 6.36 & 0.04 & -0.051 \\
\hline 684.01 & 4.03 & 0.16 & -0.280 & 1549.01 & 29.48 & 0.66 & -1.208 & 2486.01 & 4.27 & 0.02 & -0.012 \\
\hline 698.01 & 12.72 & 0.11 & -0.027 & 1560.01 & 31.57 & 0.16 & -0.220 & 2512.01 & 15.92 & 0.25 & -0.465 \\
\hline 716.01 & 26.89 & 0.06 & -0.036 & 1561.01 & 9.09 & 0.24 & -0.431 & 2513.01 & 19.01 & 0.10 & -0.183 \\
\hline 728.01 & 7.19 & 0.10 & -0.017 & 1582.01 & 186.40 & 0.08 & -0.022 & 2519.01 & 4.79 & 0.08 & -0.116 \\
\hline 772.01 & 61.26 & 0.11 & -0.107 & 1587.01 & 52.97 & 0.21 & -0.343 & 2528.01 & 12.02 & 0.05 & -0.058 \\
\hline 797.01 & 10.18 & 0.09 & -0.013 & 1591.01 & 19.66 & 0.04 & -0.006 & 2538.01 & 39.83 & 0.04 & -0.042 \\
\hline 799.01 & 1.63 & 0.06 & -0.042 & 1675.01 & 14.62 & 0.10 & -0.144 & 2572.01 & 6.38 & 0.03 & -0.001 \\
\hline 815.01 & 34.84 & 0.35 & -0.628 & 1684.01 & 62.82 & 0.06 & -0.049 & 2573.01 & 1.35 & 0.06 & -0.002 \\
\hline 833.01 & 3.95 & 0.42 & -0.791 & 1754.01 & 15.14 & 0.03 & -0.008 & 2577.01 & 18.56 & 0.20 & -0.372 \\
\hline 838.01 & 4.86 & 0.12 & -0.121 & 1761.01 & 10.13 & 0.08 & -0.110 & 2578.01 & 13.33 & 0.38 & -0.745 \\
\hline 856.01 & 39.75 & 0.14 & -0.039 & 1773.01 & 83.10 & 0.46 & -0.783 & 2639.02 & 2.12 & 0.03 & -0.024 \\
\hline 882.01 & 1.96 & 0.20 & -0.128 & 1783.01 & 134.48 & 0.08 & -0.008 & & & & \\
\hline
\end{tabular}

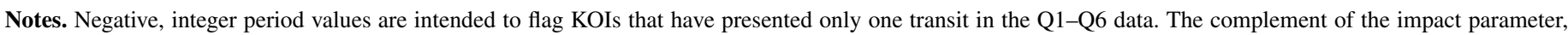

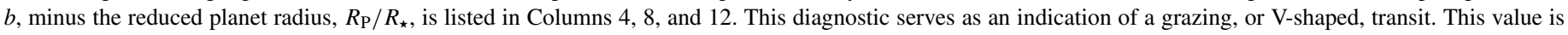

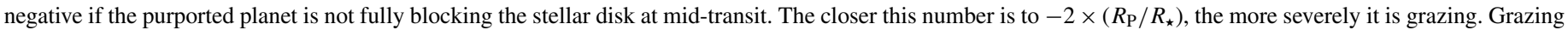
transit are required to model $\mathrm{V}$-shaped light curves.

number (SOC version number). For this analysis, Quarters 1-4 were processed with SOC 6.1 code, Quarter 5 was processed with SOC 6.2, and Quarter 6 was processed with a pre-release version of SOC 7.0. Specifics about the features of each can be found in the DRN at MAST that accompany each release. Note that quarterly data are reprocessed as new pipeline versions become available. Information about the data utilized herein can be found in DRN 4-9. We note that the Quarter 6 data archived at MAST may differ slightly from the data employed here since the latter were processed with pre-release software.

\section{TRANSIT IDENTIFICATION}

Systematic-error corrected light curves for all quarters under consideration are passed to the TPS pipeline module to identify signatures of transiting planets. The functionality of this module 
Table 3

Host Star Characteristics

\begin{tabular}{|c|c|c|c|c|c|c|c|c|c|c|c|}
\hline KOI & $\mathrm{KIC}^{\mathrm{a}}$ & $\mathrm{Kp}^{\mathrm{b}}$ & $\begin{array}{r}\mathrm{CDPP}^{\mathrm{c}} \\
(\mathrm{ppm})\end{array}$ & $\begin{array}{c}\alpha(\mathrm{J} 2000) \\
(\mathrm{hr})\end{array}$ & $\begin{array}{c}\delta(\mathrm{J} 2000) \\
(\mathrm{deg})\end{array}$ & $\begin{array}{l}T_{\text {eff }} \\
(\mathrm{K})\end{array}$ & $\log g$ & $R_{\star} / R_{\odot}$ & $M_{\star} / M_{\odot}{ }^{\mathrm{d}}$ & $f_{T_{\text {eff }}}{ }^{\mathrm{e}}$ & $f_{\mathrm{Obs}}{ }^{\mathrm{f}}$ \\
\hline 5 & 8554498 & 11.665 & 220.7 & 19.31598 & 44.6474 & 5861 & 4.19 & 1.42 & 1.14 & 3 & 111111 \\
\hline 41 & 6521045 & 11.000 & 105.5 & 19.42573 & 41.9903 & 5909 & 4.30 & 1.23 & 1.11 & 3 & 111111 \\
\hline 46 & 10905239 & 13.770 & 56.2 & 18.88370 & 48.3552 & 5764 & 4.40 & 1.10 & 1.12 & 2 & 111111 \\
\hline 70 & 6850504 & 12.498 & 198.7 & 19.17987 & 42.3387 & 5443 & 4.45 & 0.94 & 0.90 & 3 & 111111 \\
\hline 82 & 10187017 & 11.492 & 322.8 & 18.76552 & 47.2080 & 4908 & 4.61 & 0.74 & 0.80 & 3 & 111111 \\
\hline 94 & 6462863 & 12.205 & 98.8 & 19.82220 & 41.8911 & 6217 & 4.33 & 1.24 & 1.20 & 2 & 100110 \\
\hline 108 & 4914423 & 12.287 & 93.9 & 19.26564 & 40.0645 & 5975 & 4.33 & 1.21 & 1.15 & 3 & 111111 \\
\hline
\end{tabular}

Notes.

a Kepler Input Catalog number.

b Apparent magnitude in the Kepler bandpass.

${ }^{c}$ rms of Combined Differential Photometric Precision from Quarters 1-6 in units of parts per million.

d Stellar Mass is derived from surface gravity and stellar radius.

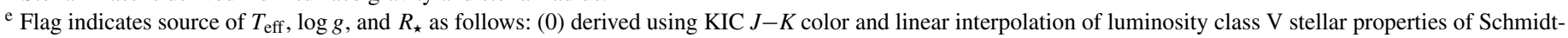

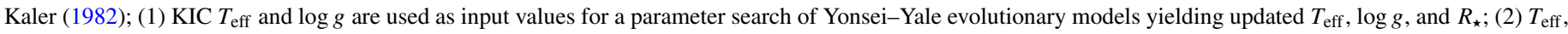

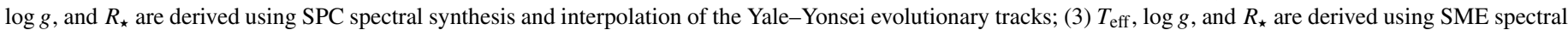
synthesis and interpolation of the Yale-Yonsei evolutionary tracks.

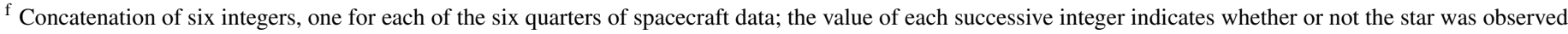

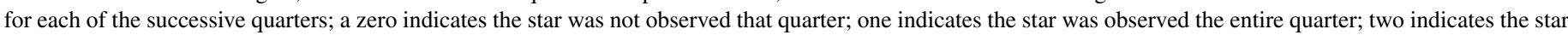

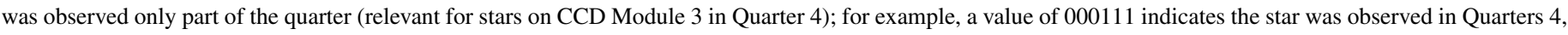
5, and 6 but not in Quarters 1, 2, or 3.

(This table is available in its entirety in a machine-readable form in the online journal. A portion is shown here for guidance regarding its form and content.)

Table 4

Planet Candidate Characteristics: Light Curve Modeling

\begin{tabular}{|c|c|c|c|c|c|c|c|c|c|c|c|c|c|c|}
\hline KOI & $\begin{array}{l}t_{\text {dur }} \\
(\mathrm{hr})\end{array}$ & $\begin{array}{l}\text { Depth } \\
\text { (ppm) }\end{array}$ & $\mathrm{S} / \mathrm{N}^{\mathrm{a}}$ & $\begin{array}{c}T_{0} \mathrm{~b} \\
\text { (days) }\end{array}$ & $\begin{array}{c}\sigma_{\mathrm{T} 0} \\
\text { (days) }\end{array}$ & $\begin{array}{c}\text { Period }^{b} \\
\text { (days) }\end{array}$ & $\begin{array}{c}\sigma_{\mathrm{P}} \\
\text { (days) }\end{array}$ & $d / R_{\star}{ }^{\mathrm{c}}$ & $\sigma_{d / R_{\star}}$ & $R_{\mathrm{P}} / R_{\star}$ & $\sigma_{R_{\mathrm{P}} / R_{\star}}$ & $b^{\mathrm{d}}$ & $\sigma_{\mathrm{b}}$ & $\chi^{2}$ \\
\hline 5.02 & 3.6882 & 20 & 8.5 & 66.36690 & 0.01456 & 7.0518564 & 0.0002848 & 9.797375 & 0.558680 & 0.00428 & 0.00038 & 0.74970 & 0.29480 & 1.4 \\
\hline 41.02 & 4.4764 & 76 & 36.8 & 66.17580 & 0.00321 & 6.8870994 & 0.0000617 & 6.177925 & 0.120880 & 0.00918 & 0.00016 & 0.86500 & 0.10100 & 1.2 \\
\hline 41.03 & 6.1426 & 92 & 23.2 & 86.98394 & 0.00667 & 35.3331429 & 0.0006257 & 18.376942 & 0.359570 & 0.01042 & 0.00030 & 0.92010 & 0.09750 & 1.2 \\
\hline 46.02 & 3.7909 & 58 & 9.7 & 65.51465 & 0.01139 & 6.0290779 & 0.0001918 & 6.892009 & 0.118930 & 0.00799 & 0.00059 & 0.83650 & 0.22630 & 1.2 \\
\hline 70.05 & 3.6029 & 99 & 18.4 & 68.20094 & 0.00566 & 19.5778928 & 0.0002980 & 28.131385 & 3.635640 & 0.00998 & 0.00039 & 0.74920 & 0.23260 & 1.1 \\
\hline$\ldots$ & $\ldots$ & $\ldots$ & $\ldots$ & $\ldots$ & $\ldots$ & & & & & & & & & \\
\hline 1612.01 & 1.2288 & 30 & 12.2 & 65.68197 & 0.00209 & 2.4649988 & 0.0000198 & 11.917724 & 4.152810 & 0.00531 & 0.00025 & 0.63910 & 0.50290 & 1.3 \\
\hline 1613.01 & 4.2342 & 78 & 22.0 & 74.08600 & 0.00466 & 15.8662120 & 0.0001989 & 18.014821 & 4.615850 & 0.00933 & 0.00064 & 0.78970 & 0.35850 & 1.0 \\
\hline 1615.01 & 1.7195 & 108 & 31.9 & 65.23335 & 0.00161 & 1.3406380 & 0.0000107 & & & & 0.00024 & 0.58290 & 0.28050 & 1.6 \\
\hline 1616.01 & 2.4240 & 147 & 23.0 & 72.41072 & 0.00363 & 13.9328148 & 0.0001269 & 41.632554 & 20.251940 & 0.01117 & 0.00119 & 0.35180 & 0.91970 & 1.2 \\
\hline 1618.01 & 3.5409 & 29 & 19.0 & 65.25658 & 0.00455 & 2.3643203 & 0.0000365 & 3.150859 & 0.318180 & 0.00547 & 0.00020 & 0.81200 & 0.17720 & 1.2 \\
\hline
\end{tabular}

Notes. Invalid and/or missing data are given values of -99 . Zero denotes a value smaller than the recorded precision.

${ }^{a} \mathrm{~S} / \mathrm{N}$ of the phase-folded transit signal computed from modeling of Quarters 1-8 data.

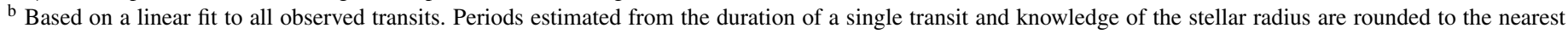
integer and multiplied by -1 .

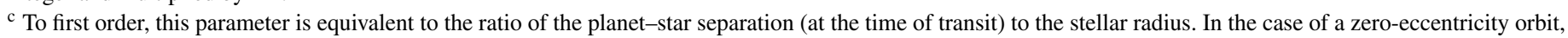
it is equivalent to the reduced semimajor axis, $a / R_{\star}$.

$\mathrm{d}$ Note that there is a strong covariance between $b$ and $d / R_{\star}$.

(This table is available in its entirety in a machine-readable form in the online journal. A portion is shown here for guidance regarding its form and content.)

is described in Jenkins et al. (2010b), Tenenbaum et al. (2012), and in the Kepler Data Processing Handbook at MAST. Before searching for transits, the software stitches together each quarterly data segment to form one contiguous light curve. To accomplish this, TPS removes a polynomial fit constrained to achieve zero offset and zero slope in the first and last day of each quarter and to ensure that the result is approximately zeromean and wide sense stationary. TPS then identifies and removes strong sinusoidal features from quarterly light curves via a periodogram-based approach. Finally, the gaps between quarters are filled via an autoregressive modeling technique to condition the time series for the Fast-Fourier-Transform-based detection algorithm.
Transit signals are identified using an adaptive, wavelet-based matched filter that explicitly takes the power spectral density (PSD) of the observation noise (stellar variability + shot noise + residual instrument noise) into account in formulating the detection statistics for each light curve. TPS transforms the time series into the wavelet domain (a joint-time/frequency analysis) in order to characterize the PSD as a function of time and then correlates a transit pulse of a given duration with the normalized, conditioned light curve in this wavelet domain to generate a correlation statistic time series that measures the likelihood that a transit is present at each time step. The single event correlation time series is then folded over each trial period from the minimum ( 0.5 days) to the length of the data set to 
Table 5

Planet Candidate Characteristics and Vetting Metrics

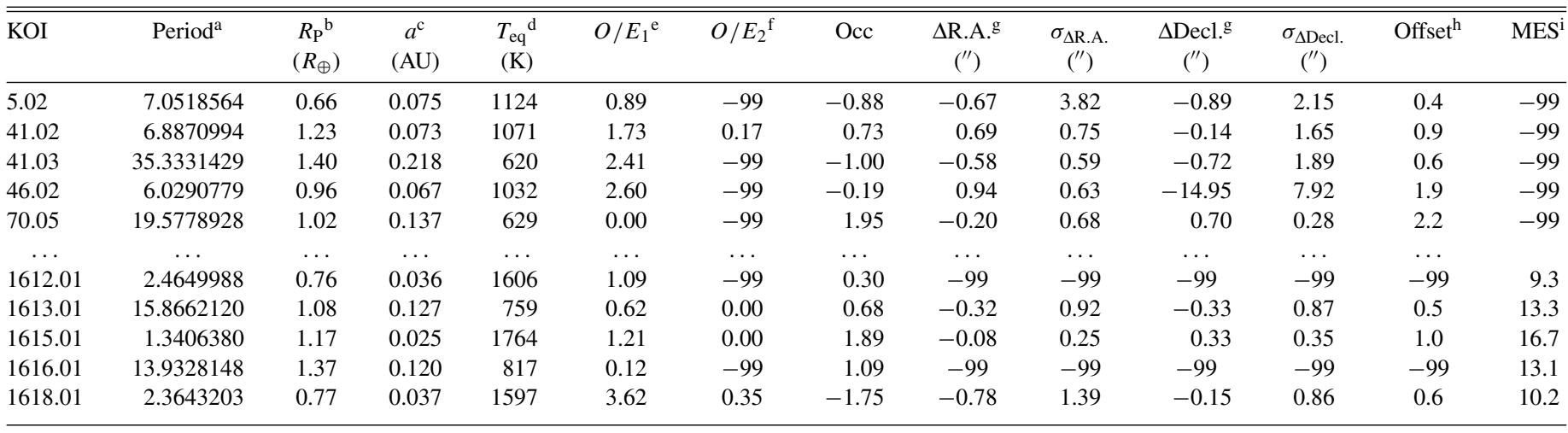

Notes. Invalid and/or missing data are given values of -99 . Zero denotes a value smaller than the recorded precision.

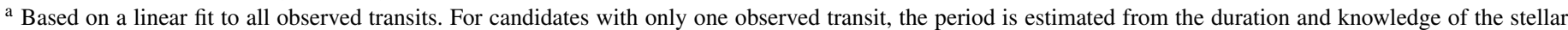
radius; values are then rounded to the nearest integer and multiplied by -1 .

b Product of $r / R *$ and the stellar radius given in Table 1.

c Based on Newton's generalization of Kepler's third law and the stellar mass in Table 3.

d See the main text for discussion.

e Odd/even statistic derived from light curve modeling.

${ }^{\mathrm{f}}$ Odd/even statistic reported by Data Validation pipeline.

g Offset is transit source position minus target star position.

${ }^{\mathrm{h}}$ Distance to source position divided by noise.

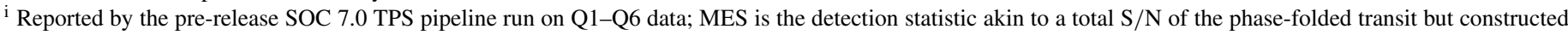
using the matched filter correlation statistics over phase and period.

(This table is available in its entirety in a machine-readable form in the online journal. A portion is shown here for guidance regarding its form and content.)

identify threshold crossing events (TCEs): instances where a given period and epoch exceed the detection threshold of 7.1 $\sigma$. Fourteen distinct transit searches are conducted, each using a different pulse duration $(1.5,2.0,2.5,3.0,3.5,4.5,5.0,6.0$, $7.5,9.0,10.5,12.0,12.5$, and $15 \mathrm{hr}$ ) as described in Jenkins et al. (2010b) and Tenenbaum et al. (2012). The maximum multiple event statistic (MES) over all durations is an estimate of the ratio of the transit depth to the uncertainty in the transit depth as a fitted parameter for the given rectangular transit pulse train at which the maximum occurred. At a threshold of $7.1 \sigma$, fewer than one false alarm is expected over the baseline mission duration due to statistical fluctuations. This defines the threshold for consideration as a viable candidate. Often, several of the matched filter pulse durations yield a detection statistic that passes our criterion (as expected), in which case the highest value is adopted. MES values are presented in Column 14 of Table 5. Two hundred ninety-one candidates are assigned MES values of -99 signifying an invalid value. This occurs when the period returned by the pipeline is not the final period derived from full light curve modeling. ${ }^{31}$ MES values of -99 also occur for the new "multis" (additional candidates associated with stars already having at least one candidates) identified by non-pipeline products (see below).

The analysis presented here stems from a TPS run used for verification and validation of the pre-release SOC 7.0 pipeline. It was the first time that TPS was run using the multiquarter functionality. Such functionality was not available for the production of the B11 catalog. Long-period candidates in the B11 catalog were identified using a box least-squares (BLS) algorithm (see, for example, Kovács et al. 2002). The BLS frequency spectrum is normalized by a smoothed time-series

\footnotetext{
31 TPS usually gets the correct period for single transit signatures but occasionally chooses a multiple of the true period as the maximum, and is sometimes confused by light curves with multiple transiting planet signatures.
}

periodogram in order to remove the $1 / f$ noise floor which can dominate the detection statistic when searching for long-period events.

TPS returned 104,999 unique targets with at least one sequence of periodic transits yielding MES > 7.1 (i.e., TCEs). The majority of these TCEs are triggered by transients in the normalized light curves. Hence, the list is further culled by applying a second criterion based on the ratio of the MES to the maximum single event statistic (SES) contributing to the MES. The SES is the maximum correlation statistic in the time domain at a given test period. MES/SES should be comparable to the square-root of the number of observed transits. Tenenbaum et al. (2012) shows that there are two distinct populations of TCEs, with a dividing line at MES $/ \mathrm{SES}=\sqrt{2}$. Below this value, detections are likely the result of two highly unequal single events as opposed to two legitimate transits of equal depth and duration. Discarding TCEs with MES/SES $<\sqrt{2}$ reduces the number of viable TCEs from 104,999 to 4531.

A transit model (Mandel \& Agol 2002) is applied to all 4531 remaining TCEs, and the results are manually inspected to identify obvious false alarms and other astrophysically interesting signals that are clearly not consistent with the planet interpretation. Examples include instrumental flux outliers that persist through the pipeline's systematic error correction modules, obvious eclipsing binary star systems, pulsating stars, and magnetically active, rapidly rotating stars. The result is a list of 1058 stars that were then assigned Kepler Object of Interest (KOI) numbers. All 1058 stars, as well as those reported in the B11 catalog, were searched for evidence of additional transit sequences. The search was performed by subtracting the transit model for the primary TCE, and then passing the residual to the modified BLS transit detection software. This yielded an additional list of 332 candidates associated with known KOIs. As in Borucki et al. $(2011 \mathrm{~b})$, decimal values $(.01, .02, .03, \ldots)$ are added to 
the KOI number to distinguish between multiple candidates associated with the same star. They are assigned in the order they were identified.

Future pipeline runs include improvements that increase the detection efficiency of multiple planet systems thereby eliminating the need to run offline tools and increasing sample uniformity.

\section{CANDIDATE VETTING}

The procedures described in Section 3 produce 1390 KOIs that are then vetted for astrophysical false positives in the form of eclipsing stellar systems. Here, we describe the suite of statistical tests employed. They are separated by the type of data they operate on. Statistical tests derived from the flux time series and the corresponding transit models are described in Section 4.1, while statistical tests derived from pixel-level data are described in Section 4.2. Both make use of pipeline products as well as offline analyses. The pipeline module that evaluates KOIs for likely false positives, referred to as Data Validation (DV), is described by Wu et al. (2010) and the Kepler Processing Handbook at MAST. Section 4.3 describes the overall procedures that were followed to promote a KOI to planet candidate status.

As work to identify and vet candidates progressed, new products became available. Quarter 7 and Quarter 8 photometry, for example, was available in the summer of 2011. These data were utilized in the offline (i.e., non-pipeline) light curve modeling used to determine various vetting metrics, as well as the properties of the planet candidates tabulated in Tables 4 and 5 and described Section 5.1. Moreover, testing of pre-release SOC 8.0 code (also using Q1-Q8 data) in the fall of 2011 produced significantly improved DV reports and metrics and were also used to vet the Q1-Q6 candidates reported in this contribution.

A subset of the vetting metrics used for candidate evaluation are provided in Table 5 so that users can identify the weaker candidates and know what types of problems to look for. These metrics are described in turn below and summarized in Section 4.3.

\subsection{Tests on the Flux Time Series}

For each KOI, the even-numbered transits and odd-numbered transits are modeled independently using the techniques described in Section 3. The depth of the phase-folded, evennumbered transits is compared to that of the odd-numbered transits as described in Batalha et al. (2010a) and Wu et al. (2010). A statistically significant difference in the transit depths is an indication of a diluted or grazing eclipsing binary system. A similar metric is computed by the DV pipeline module as described by Wu et al. (2010). Each uses a different methodology for detrending the light curves (i.e., filtering out stellar variability), and both proved useful and are tabulated in Columns 6 (modeling-derived statistic: $\left.O / E_{1}\right)$ and 7 (DV-derived statistic: $O / E_{2}$ ) of Table 5. In general, $3 \sigma$ was the threshold for flagging a KOI as a false positive. However, when the two values disagreed, we deferred to the DV statistic owing to its more sophisticated whitening filters. One hundred forty-three KOIs in Table 5 have $O / E_{1}>3$ while only seven have $O / E_{2}>3$. Five have both $O / E_{1}$ and $O / E_{2}$ larger than $3 \sigma$ but are otherwise clean candidates. Further inspection of their light curves suggested that stellar variability and/or instrumental transients were driving an anomalously high odd/even statistic, and the candidates were retained. In 121 cases, the DV model fitter failed, thereby precluding quantification of an odd/even statistic. In 18 of these cases, $O / E_{1}$ is larger than $3 \sigma$ and the candidate was retained anyway. While most of these are marginal cases near the $3 \sigma$ cutoff, users are cautioned that exceptions exist and should be examined independently on a case-by-case basis.

The modeling allows for the presence of a secondary eclipse (or occultation event) near phase $=0.5$ as a means of identifying diluted or grazing eclipsing star systems. The Secondary Statistic (Column 8 of Table 5) is the relative flux level at phase 0.5 , divided by the noise. As such, it can have positive as well as negative values. While its presence does not rule out the planetary interpretation, it acts as a flag for further investigation. More specifically, the flux decrease is translated into a surface temperature assuming a thermally radiating disk, and this temperature is compared to the equilibrium temperature of a low-albedo (0.1) planet at the modeled distance from the parent star. If the flux change is not severe enough to rule out the planetary interpretation (ascertained by the difference between the surface temperature and equilibrium temperature), the candidate is retained. Eight KOIs retained in the catalog have a statistic outside of (negative) $3 \sigma$. In each of these cases, it appears possible that the occultation signal is a result of stellar and/or instrumental flux changes. This statistic is relevant primarily for short-period orbits where circularization is expected since the search is only done at phase 0.5 . The DV pipeline module checks to see if additional transit sequences were identified in the light curve at the same period but different phase. No such sequences were identified for the candidates reported here.

Table 2 lists KOIs (both old and new) that are $V$-shaped. The shape of a transit is not used as a diagnostic for rejecting planet candidates. The right combination of properties and geometry can, indeed, produce ingress and egress times that are a significant fraction of the total transit duration (e.g., grazing transits). However, a diluted eclipsing binary system is another possible interpretation and does not require such a narrow range of inclination angles (impact parameters). The false-positive rate amongst the $V$-shaped candidates is expected to be higher than the false-positive rate of the general population. A metric is constructed to flag such cases: $1-b-\left(R_{\mathrm{P}} / R_{\star}\right)$. The objective is to alert the reader to populations with larger false-positive rates and also to avoid classification of a transit as $V$-shaped because of smear produced by the 30 minute cadence. Errors in the metric are dominated by the uncertainty of the modeled impact parameter which can be quite high. Therefore, all flagged light curves were visually inspected.

Negative values imply that the purported planet is not fully covering the stellar disk at mid-transit. The closer the metric is to $-2 \times\left(R_{\mathrm{P}} / R_{\star}\right)$, the more severely it is grazing. A grazing geometry is required to model $V$-shaped transits that are not caused by a large planet-to-star size ratio. The KOIs with light curves modeled as grazing transits are listed in Table 2 together with the orbital period and reduced radius.

\subsection{Tests on the Pixel Data}

A major source of false-positive planetary candidates in the Kepler data is a background eclipsing binary (BGEB) star within the photometric aperture of a Kepler target star. These BGEBs, when diluted by a target star, can mimic a planetary transit signal. Two methods are used to detect such BGEBs by using the Kepler pixels to determine the location of the object causing the transit signal: direct measurement of the source location via difference image analysis and inference of the source location from photo-center motion associated with the transits. 

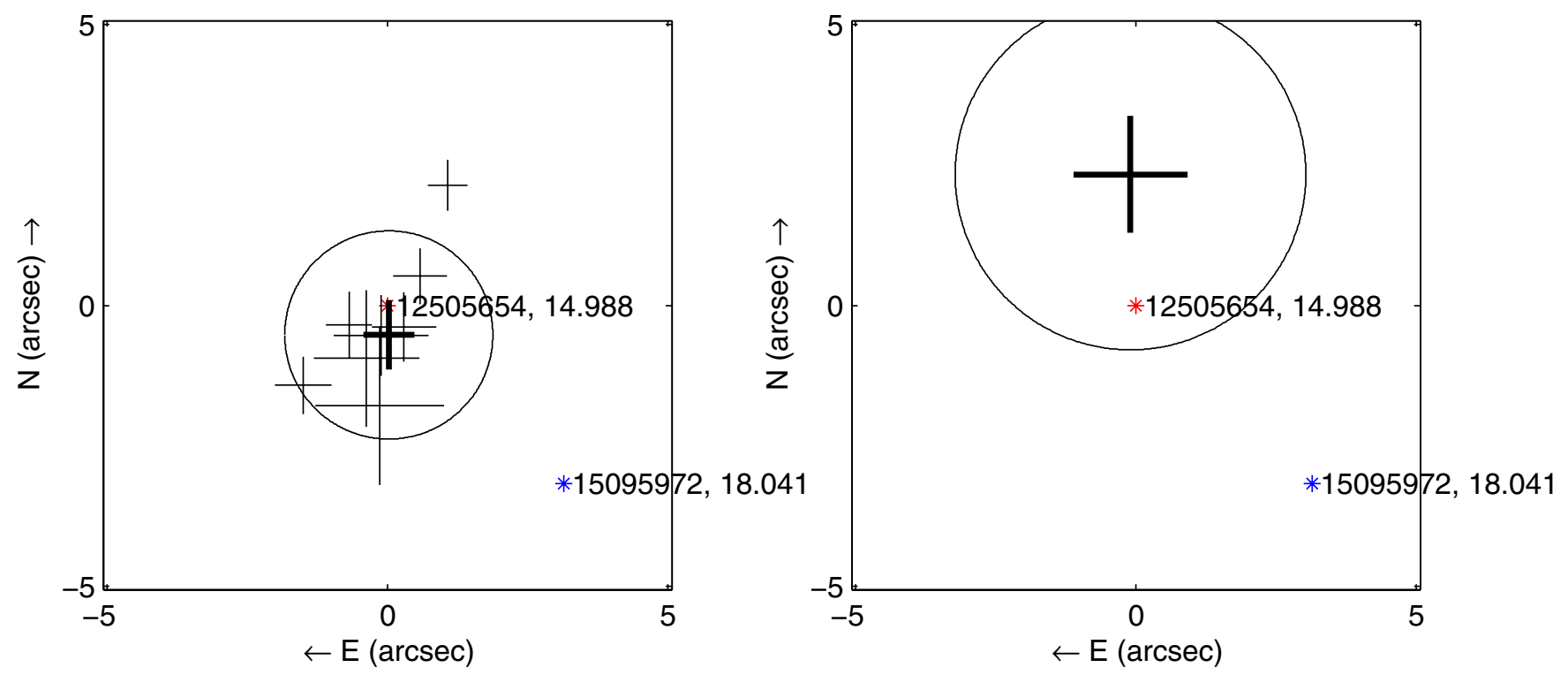

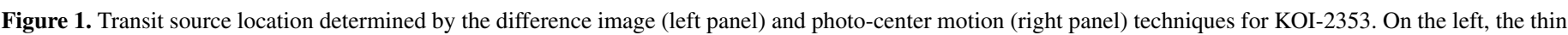

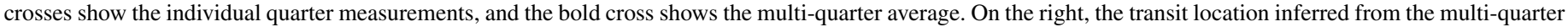

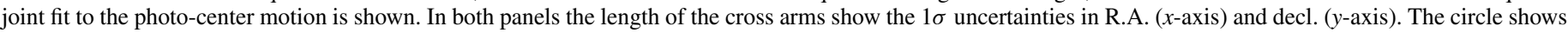

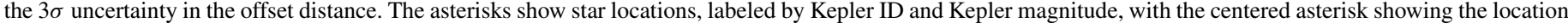
of the target star. This is a clear example where the transits are associated with the target, rather than the nearby, faint background star.

(A color version of this figure is available in the online journal.)

Photo-center motion is measured by computing the fluxweighted centroid of all pixels downlinked for a given star both in and out of transit. Fluxes associated with a transit event are identified using the ephemerides and transit durations listed in Table 2. The flux-weighted centroids are calculated in one of two ways. KOIs that were processed with the DV module in the SOC pipeline have a flux-weighted centroid measurement for every cadence. The in versus out of transit centroid offset is computed by performing a least-squares fit of the pipelinegenerated transit model to the multi-quarter centroid time series, the idea being that the behavior of the flux-weighted centroid time series will mirror that of the flux time series. The centroid offset is the amplitude of the "transit" pulse in the model fit.

KOIs that were not processed by the DV pipeline module are treated slightly differently in that the flux-weighted centroid is computed for a pixel image constructed by taking the average of images in a single quarter when the KOI is not transiting. It is then compared to the flux-weighted centroid for a pixel image that is the average of images in the same quarter when the KOI is transiting. The centroid offset is the difference between the in and out of transit centroids. Quarterly offsets are averaged together as described below. Once the centroid offset is computed, the source location is inferred by scaling it by the inverse of the flux as described in Jenkins et al. (2010a).

Difference image analysis takes the difference between average in-transit pixel images and average out-of-transit images. Barring pixel-level systematics and field-star variability, the pixels with the highest flux in the difference image form a star image at the location of the transiting object, with flux level equal to the fractional depth of the transit times the original flux of the star. Performing a fit of the Kepler pixel response function (PRF; Bryson et al. 2010) to both the average difference and out-oftransit images gives the sky location of the transit source and the target star. The offset of the transit source from the target star is then defined as the transit source minus target star location. For most KOIs, the difference image offset is computed per quarter, and the quarterly offsets are averaged as described below. For a small number of KOIs with very low signal-to-noise ratio $(\mathrm{S} / \mathrm{N})$, a computationally expensive joint multi-quarter fit is performed, with uncertainties estimated via a bootstrap analysis.

In principle, both the photo-center motion and difference image techniques are similarly accurate for isolated stars and sufficiently high $\mathrm{S} / \mathrm{N}$ transits, but the techniques have different responses to systematic error sources such as field crowding. The photo-center method is more sensitive to noise for low-S/N transits and crowding by field stars. In particular, the estimate of the transit source location by scaling the offset is highly sensitive to incompletely captured flux for either the target star or field stars in the aperture.

In the difference image method, the PRF fit to the difference and out-of-transit pixel images is biased by PRF errors described in Bryson et al. (2010), as well as errors due to crowding. Defining the centroid offset as the difference between the outof-transit and difference images nearly cancels the PRF bias because both fits are subject to the same error. Bias due to crowding, however, is more of an issue. Excepting cases in which background stars are strongly varying, a difference image removes most point sources, leaving the transit signal as the predominant change in flux. The average out-of-transit image, on the other hand, contains all point sources. Consequently, the offset (formed by comparing a direct image and a difference image) may contain a bias.

Both bias types vary from quarter to quarter. A study of a large number of targets indicates that the combined biases have an approximately zero-mean distribution across quarters, so we can reduce their impact by averaging the quarterly centroid measurements across quarters. We compute this average by performing a robust $\chi^{2}$ minimizing fit to the quarterly results. This approach produces an uncertainty that takes into account both the uncertainty of the individual quarterly observations as well as their scatter due to bias across quarters. An example set of quarterly measurements and the resulting average is shown in the left panel of Figure 1. This method works best for shortperiod candidates, where there are many transits in all quarters. 
This method is less effective in reducing the centroid bias for long-period candidates. In particular, single-transit candidates that appear in only one quarter may have unknown biases in their centroid positions that are not accounted for in the centroid uncertainties.

The above centroid analysis was performed using data from Quarters 1-8. The transit source location offsets reported in Table 5 are from the difference image method since it is more reliable as evidenced by consistently smaller uncertainties compared to the photo-center motion method. A candidate passes the photo-center vetting step when its multi-quarter transit source offset is less than $3 \sigma$. There are, however, exceptions. KOIs having transit source offsets larger than $3 \sigma$ were identified as having systematic errors (e.g., crowding biases) that are likely causing the large apparent offset. Modeling efforts to confirm these systematics are currently underway.

In some cases, the PRF fit algorithm failed, typically due to low quarterly $\mathrm{S} / \mathrm{N}$ or to bright field stars that prevented reliable determination of the target star location via PRF fit. We retain these targets when visual inspection of the difference image indicates that the change in flux due to the transit is on the target star. Finally, difference imaging is very inaccurate for saturated targets, and we retain saturated candidates for which the difference images show no obvious indication of a background source. Offset values for slightly saturated stars (Kp between 10.5 and 11.5) are likely accurate to within $4^{\prime \prime}$ (one pixel), while offset values for highly saturated stars $(\mathrm{Kp}<10.5)$ should be disregarded. Transit source offset values are set to -99 when we feel that the centroid measurement is unreliable.

\subsection{Promotion to Planet Candidate}

KOIs were divided amongst more than 20 science team members for evaluation of the following metrics: (1) odd/even statistic, (2) occultation test, (3) quality of model fit, (4) long/short period comparison, (5) single-quarter photocenter motion, and (6) multi-quarter photo-center motion. An integer value of 0,1 , or 2 was assigned to each metric to indicate if the test clearly passed (0), was ambiguous (1), or clearly failed (2). A similar flag was ascribed to the visual appearance, where examples of suspicious characteristics would include markedly V-shaped transits, red noise and/or outliers in the time series calling into question the reliability of the transit signal, anomalously long transit durations, poor light curve fits, obvious secondaries, etc. Each candidate was then designated "yes," "no," or "maybe." Candidates flagged as ambiguous (maybe) were re-evaluated. In most cases, this required updated light-curve modeling, more detailed inspection of the software pipeline products, and/or further scrutiny of the pixel flux analysis yielding photo-center statistics.

Once every KOI was assigned a "yes" or "no" designation, the integer flags were summed and the distribution for the two populations was compared. This elucidated a small number $(<10)$ of inconsistencies in which flags indicate a problem yet the KOI was assigned a "yes" designation. These were independently evaluated.

The period and location on the sky of every new KOI was cross-checked against the list of previously known KOIs and the catalog of eclipsing binaries (Prša et al. 2011; Slawson et al. 2011). This is a safeguard against redundancy. More importantly, though, the cross-check serves to identify flux contamination from bright eclipsing binaries that the photocenter analysis missed. Approximately 25 targets within $20^{\prime \prime}$ of an existing KOI or eclipsing binary with commensurate (within a factor of $1,2,3$ ) orbital period (to within 5 minutes) were identified. In this manner, we also identified two small swarms of spatially co-located stars, each with a transit-like event at a commensurate orbital period. The lack of sizable photo-center motion and the spatial extent of the contaminating flux suggests that scattered light (e.g., an optical ghost from a bright eclipsing binary at the focal plane anti-podal position) is responsible.

Every one of the 1390 KOIs evaluated here will appear either in this contribution as a viable planet candidates, or in the associated false-positive catalog (S. Bryson et al. 2012, in preparation), or in future versions of the Eclipsing Binary Catalog.

Eight high-S/N $(>30 \sigma)$ single transit events were identified and included in the catalog. Their corresponding orbital periods are estimated from the transit duration and knowledge of the stellar radius assuming zero eccentricity. The periods are then rounded to the nearest integer and multiplied by -1 so as to distinguish them from the candidates that have reliable orbital ephemerides. Parameters requiring an accurate period (e.g., odd/even statistic, semimajor axis, etc.) are set to -99 , which is the value adopted globally for unreliable, spurious, or unknown values.

The final list of viable planet candidates is presented in Table 4 with additional information listed in Table 5. In addition to the MES from the Q1-Q6 TPS run, the following vetting metrics are provided: (1) the odd/even statistic that tests for an eclipsing star system of nearly equal-mass components at twice the period (Columns 6 and 7), (2) the occultation (secondary) statistic that tests for a weak secondary event inconsistent with a planet occultation at phase 0.5 (Column 8), (3) the photo-center offsets in R.A. and decl. measured in arcsec and their associated uncertainties (Columns 9-12), and (4) the total photo-center offset position measured in units of the noise (Column 13).

Analysis is based on a blend of both quantitative metrics and manual inspection. Both the promotion from TCEs to KOIs and the promotion of KOIs to planet candidates has a human element that not only increases the reliability of the catalog but also reduces the number of false negatives that are discarded. The reader is cautioned, however, that reliability and efficiency of human scrutiny is difficult to quantify. Consequently, sample statistics derived from this sample will be difficult to interpret. This will improve with each subsequent catalog release as we work toward automation and uniformity of procedures and metrics.

\section{PROPERTIES OF PLANET CANDIDATES}

Here, we describe the light curve modeling that characterizes both the orbital and physical characteristics of each planet candidate.

\subsection{Model Fitting}

For each KOI, a transit model was fit to the data. The transit model uses the analytic formulae of Mandel \& Agol (2002) to model the transit and a Keplerian orbit to model the orbital phase. The model fits for the mean stellar density $\left(\rho_{\star}\right)$, center of transit $\left(T_{0}\right)$, orbital period $(P)$, scaled planetary radius $\left(R_{\mathrm{P}} / R_{\star}\right)$, impact parameter $(b)$, and occultation (secondary) depth (at phase $=0.5$ ). In the case of multiple transiting candidates the orbits were assumed to be non-interacting and $T_{0}, P, b$ and the occultation depth were fit for each candidate. The assumption is made that all candidates in the same system orbit the same star modeled by $\rho_{\star}$ and that the total mass 
of the companion is much less than the mass of the central star. For a Jupiter-mass companion, an error of $0.02 \%$ will be incurred on the measurement of $\rho_{\star}$, a $0.1 M_{\odot}$ companion would skew our estimate of $\rho_{\star}$ by approximately $2 \%$, and a $0.5 M_{\odot}$ companion would induce a systematic error of $41 \%$ on $\rho_{\star}$. These assumptions give

$$
\left(\frac{a}{R_{\star}}\right)^{3} \frac{\pi}{3 G P^{2}}=\frac{\left(M_{\star}+M_{\mathrm{p}}\right)}{\frac{4 \pi}{3} R_{\star}^{3}} \approx \frac{M_{\star}}{\frac{4 \pi}{3} R_{\star}^{3}}=\rho_{\star},
$$

where $a / R_{\star}$ is referred to as the reduced semimajor axis. Transits probe a small portion of an orbit giving little or no information about eccentricity. We assume circular orbits in our models. Consequently, the reduced semimajor returned by light curve modeling does not necessarily yield an accurate determination of the semimajor axis nor the stellar density. To emphasize this point, we purposely report the parameter as $d / R_{\star}$ which, to first order, is equivalent to the ratio of the planet-star separation during transit to the stellar radius. It is equivalent to $a / R_{\star}$ for zero-eccentricity orbits.

A full orbital solution is required to correctly determine stellar parameters from transit modeling. Great care must be taken when interpreting the fitted stellar parameters. A mismatch between the reported stellar parameters and a transit-derived stellar parameter can be interpreted as evidence of: an eccentric orbit, erroneous stellar classification, or a transiting companion with significant mass. One is not able to distinguish among these three cases using the model parameters provided. Best-fit model parameters were determined by computing a chi-square minimization search using a Levenberg-Marquardt algorithm (Press et al. 1992). Derivatives were numerically determined. Uncertainties are taken from the diagonal elements of the formal covariance matrix corresponding to each fitted parameter. They do not account for correlated errors between parameters.

\subsection{Stellar Parameters}

Output parameters from light curve modeling can be used to compute the radius, semimajor axis, and equilibrium temperature of each planet candidate given knowledge of the stellar surface temperature, radius, and mass. These and other stellar properties for the host stars are listed in Table 3. Stellar coordinates and the apparent magnitude in the Kepler bandpass are obtained from the KIC at MAST (Brown et al. 2011).

The KIC has been a valuable resource for the stellar properties necessary for characterizing Kepler's planet candidates (e.g., $T_{\text {eff }}, \log g$, and $R_{\star}$ ). However, there are parameters in the KIC that imply populations that are inconsistent with stellar theory and observation. For example, G-type stars with $\log g \approx 5$ (i.e., well below the main sequence) are not uncommon in the KIC. While the uncertainty on the determination of $\log g$ shows that such a star is consistent with being near the main sequence, using such stellar parameters results in small stellar and planetary radii and an underestimation of incident flux on the planet candidate. We systematically correct such populations in the KIC by matching $T_{\text {eff }}, \log g$ and $[\mathrm{Fe} / \mathrm{H}]$ to the Yonsei-Yale stellar evolution models (Demarque et al. 2004). We find the closest match based on minimization of

$$
\chi^{2}=\left(\frac{\delta T_{\text {eff }}}{\sigma_{T_{\text {eff }}}}\right)^{2}+\left(\frac{\delta \log g}{\sigma_{\log g}}\right)^{2}+\left(\frac{\delta[\mathrm{Fe} / \mathrm{H}]}{\sigma_{[\mathrm{Fe} / \mathrm{H}]}}\right)^{2},
$$

where $\delta$ represents the difference in the KIC and Yonsei-Yale parameter and $\sigma$ is the adopted uncertainty in the KIC parameter.

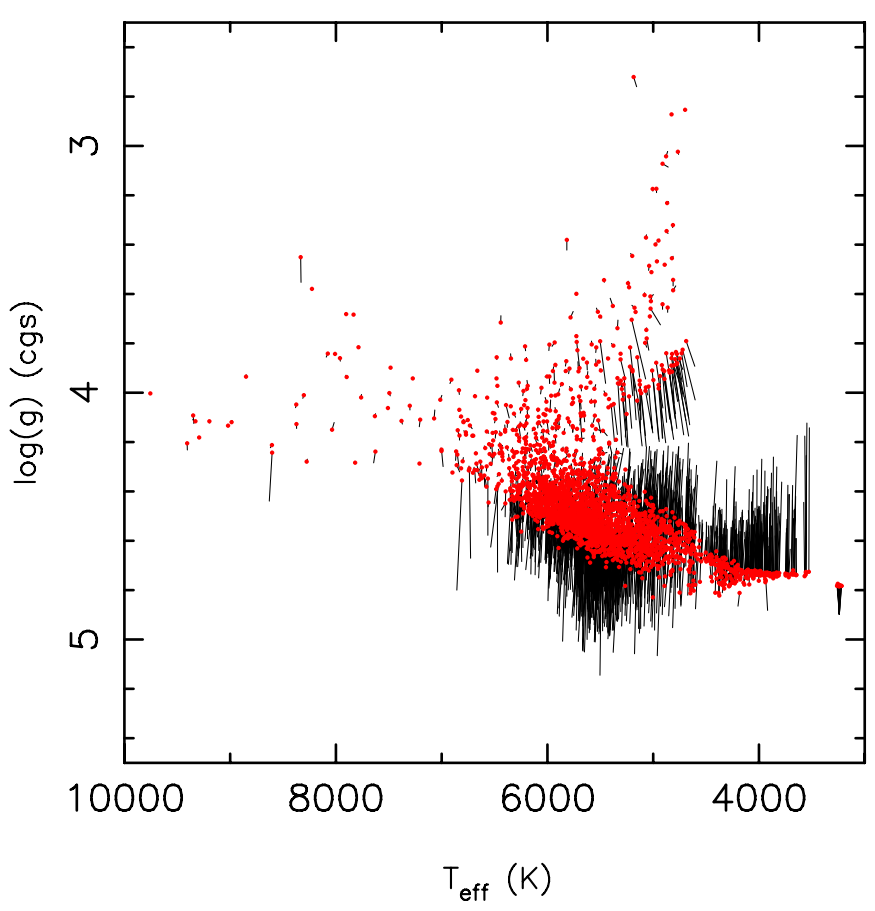

Figure 2. Surface gravity vs. effective temperature for the host stars. The red dots correspond to updated values based on a search of the Yonsei-Yale evolution models. Black lines point back to the locations defined by the KIC values.

(A color version of this figure is available in the online journal.)

We adopted $\sigma_{T_{\text {eff }}}=200 \mathrm{~K}, \sigma_{\log g}=0.3$, and $\sigma_{[\mathrm{Fe} / \mathrm{H}]}=0.4$ and required the modeled age to be less than 14 Gyr. For each star we adopt the model-determined mass and radius. Figure 2 shows $T_{\text {eff }}$ and $\log g$. The red dots show updated values that have been adopted for determination of the stellar mass and radius. Stellar properties derived in this manner are flagged in Table 3 by setting $f_{\mathrm{T}_{\text {eff }}}$ equal to 1 . The black lines point to the star's original location based on the KIC.

There are three populations that show significant changes to the determined $\log g$. (1) Stars that fall below the main sequence move to smaller values of $\log g$. These stars have $T_{\text {eff }}$ from roughly $4500 \mathrm{~K}$ to $6500 \mathrm{~K}$. In general, estimates of the properties of these stars become larger and more luminous, which reduces the number of small stars and increases the amount of incident flux on the orbiting companion. (2) Most stars cooler than $4500 \mathrm{~K}$ see a substantial decrease in $\log g$. However, modeled masses and radii are highly uncertain in this temperature range and should be used with caution. (3) There is a population of stars which have KIC $\log g$ near 4.1 and $T_{\text {eff }}$ near $5000 \mathrm{~K}$. Stars in this region have no match when we restrict model ages to $\leqslant 14$ Gyr. Such stars either move toward the red giant branch (lower values of $\log g$ ) or toward the sub-giant branch (larger values of $\log g$ ).

Forty-nine host stars listed in Table 3 have revised stellar properties determined from high-resolution spectroscopy taken as part of the Kepler ground-based follow-up observation program using the Shane $3 \mathrm{~m}$ telescope at Lick Observatory, the $1.5 \mathrm{~m}$ Tillinghast reflector at Whipple Observatory, the $2.7 \mathrm{~m}$ Harlan Smith telescope at McDonald, the $2.5 \mathrm{~m}$ Nordic Optical Telescope at La Palma, Spain, and the $10 \mathrm{~m}$ Keck telescope using HIRES. Fourteen of these were subjected to LTE spectral synthesis using Spectroscopy Made Easy (SME) (Valenti \& Piskunov 1996; Valenti \& Fischer 2005). The resulting surface gravity, effective temperature, and metallicity are used to identify the best-matching Yonsei-Yale stellar 
evolution model (Demarque et al. 2004) as described above for the KIC parameter adjustments. Stellar properties derived in this manner are flagged in Table 3 by setting $f_{\mathrm{T}_{\text {eff }}}$ equal to 3 .

The spectra of 45 host stars were compared to a synthetic library of spectra as part of the Stellar Parameter Classification (SPC) effort and analysis tool described by Buchhave et al. (2012). As in the case of the SME analysis, the resulting stellar parameters are compared to the Yonsei-Yale models to determine the stellar radius. The revised stellar properties are listed in Table 3 and flagged with $f_{\mathrm{T}_{\text {eff }}}$ equal to 2 .

Twenty-one host stars have neither KIC classifications nor spectroscopically derived stellar properties. In these cases $\left(f_{\mathrm{T}_{\text {eff }}}\right.$ equal to 0 ), the effective temperature is estimated from linear interpolation of the main-sequence properties of Schmidt-Kaler (1982) at the KIC $J-K$ color. There is little justification for the assumption of a main-sequence luminosity class. False positives and large errors in the planet candidate radius should be expected amongst this sample.

Also included in Table 3 is the rms Combined Differential Photometric Precision (CDPP) - a measure of the photometric noise (including stellar and instrumental sources) on a $6 \mathrm{hr}$ timescale after systematic-error correction and removal of strong sinusoidal features. A detailed description of the CDPP can be found in Jenkins et al. (2010b). CDPP values are crucial for statistical analyses of planet occurrence rates as they define the observational detection sensitivities. 3, 6, and $12 \mathrm{hr} r m s$ CDPP values for all observed stars are archived at MAST and can be obtained using the Data Retrieval Search form.

The objective of the Kepler mission is to determine planet occurrence rate as a function of planet radius and orbital period. This requires accurate stellar properties not only of the planet-hosting stars but also of the parent population of Kepler target stars. The latter is required for sensitivity corrections. By including corrections to the properties of planet-hosting stars (or subsets thereof) and not the parent sample, we are introducing a non-uniformity that can negatively impact statistical studies.

Independent analyses of systematic errors in the KIC have begun to appear in the published literature. Muirhead et al. (2012a), for example, utilize medium-resolution $K$-band spectroscopy to asses systematic errors in $T_{\text {eff }}, \log g$, and $[\mathrm{Fe} / \mathrm{H}]$ for late-K and M-type dwarfs amongst the sample of planet-hosting stars and report systematically overestimated stellar radii. Mann et al. (2012) perform a similar study of 382 target stars using medium-resolution optical spectra and show that the majority of bright $(\mathrm{Kp}<14)$ and cool $\left(T_{\text {eff }}<4500 \mathrm{~K}\right)$ stars in the Kepler target catalog are giants. Pinsonneault et al. (2012) report on systematic errors in the griz photometry in the KIC used to determine $T_{\text {eff }}, \log g$, and $R_{\star}$ among other parameters. These errors lead to underestimates in effective temperature.

The star properties listed in Table 3 do not contain corrections from these independent analyses. However, there is a concerted effort to devise a sensible strategy affecting future catalog releases-one that produces accurate estimates of star and planet properties while preserving uniformity for the purpose of statistical studies. A Kepler Star Properties Working Group has formed for this express purpose. ${ }^{32}$

In the meantime, we have checked for potential contamination of giants in the KOI sample using asteroseismology, which provides an effective means of identifying giant stars using Kepler data through the detection of solar-like oscillations

\footnotetext{
32 Information about all Kepler working groups and how to participate can be
} found at http://keplerscience.arc.nasa.gov. (see, e.g., Gilliland et al. 2010). Oscillation frequencies scale with effective temperature and surface gravity, and generally range from periods of a few minutes for main-sequence stars to several hours and longer for red giants (e.g., De Ridder et al. 2009; Chaplin et al. 2010). Red giants with $\log g \lesssim 3.5$ and $T_{\text {eff }} \lesssim 5000 \mathrm{~K}$ oscillate at frequencies $\lesssim 300 \mu \mathrm{Hz}$, and hence oscillations for these stars are readily detectable with LC Kepler data.

A preliminary analysis of all KOIs in the candidate catalog using Q1-Q10 LC data yields only one detection of giantlike oscillations in a KOI classified as a main-sequence star: KOI-2548. However, there is a nearby foreground star approximately 4 mag brighter that has the same oscillation pattern as KOI-2548 and is classified as a giant in the KIC. Highresolution spectroscopy of KOI-2548 acquired with the HIRES spectrograph on the Keck telescope is consistent with a dwarf. The analysis confirms that the majority of KOI host stars are on or near the main sequence.

Note that there may be giants that have escaped detection due to close binary interactions suppressing the oscillations (Derekas et al. 2011), but we suspect the number of such systems in the KOI catalog to be small. For stars with $T_{\text {eff }} \lesssim 4000 \mathrm{~K}$, the oscillation periods of potential giants would be too long to be sufficiently resolved with the amount of data currently available. Hence, the small number of KOIs in this temperature regime, particularly those with $\mathrm{Kp}<14$, should be viewed with caution. An in-depth asteroseismic study of KOIs using both short-cadence and long-cadence data will be presented in forthcoming papers.

\subsection{Derived Parameters}

The planet radius, semimajor axis, and equilibrium temperature of each planet candidate are computed using the estimated stellar properties and the parameters returned by the light curve modeling described in Section 5.1. Planet radius (Column 3 of Table 5) is the product of the reduced radius, $R_{\mathrm{P}} / R_{\star}$ (Column 11 in Table 4), and the stellar radius in Column 9 of Table 3. Planet radii are given in units of Earth radii.

The semimajor axis provided in Column 4 of Table 5 is derived from Newton's generalization of Kepler's third law given the orbital period (Column 2) and the stellar mass (Column 10 of Table 3). The stellar mass is derived directly from the surface gravity and stellar radius (Columns 8 and 9 of Table 3). Although the parameter $d / R_{\star}($ Column 9) is related to the reduced semimajor axis $\left(a / R_{\star}\right)$ as described in Section 5.1, the two are only equivalent for the case of a circular orbit.

The equilibrium temperature, $T_{\text {eq }}$ (Column 5 of Table 5) is the temperature at which the incident stellar flux balances the thermal radiation. It is derived by assuming that the planet and star act as gray bodies in equilibrium and that the heat is evenly distributed from the day to night side of the planet (e.g., a planet with an atmosphere or a planet with rotation period shorter than the orbital period):

$$
T_{\text {eq }}=T_{\text {eff }}\left(R_{\star} / 2 a\right)^{1 / 2}\left[f\left(1-A_{\mathrm{B}}\right)\right]^{1 / 4},
$$

where $T_{\text {eff }}$ and $R_{\star}$ are the effective temperature and radius of the host star, the planet at distance $a$ with a Bond albedo of $A_{\mathrm{B}}$. The factor, $f$, acts as a proxy for atmospheric thermal circulation where $f=1$ (assumed here) indicates full thermal circulation. The Bond albedo, $A_{B}$, is the fraction of total power incident on a body scattered back into space, which we assume to be $30 \%$. 


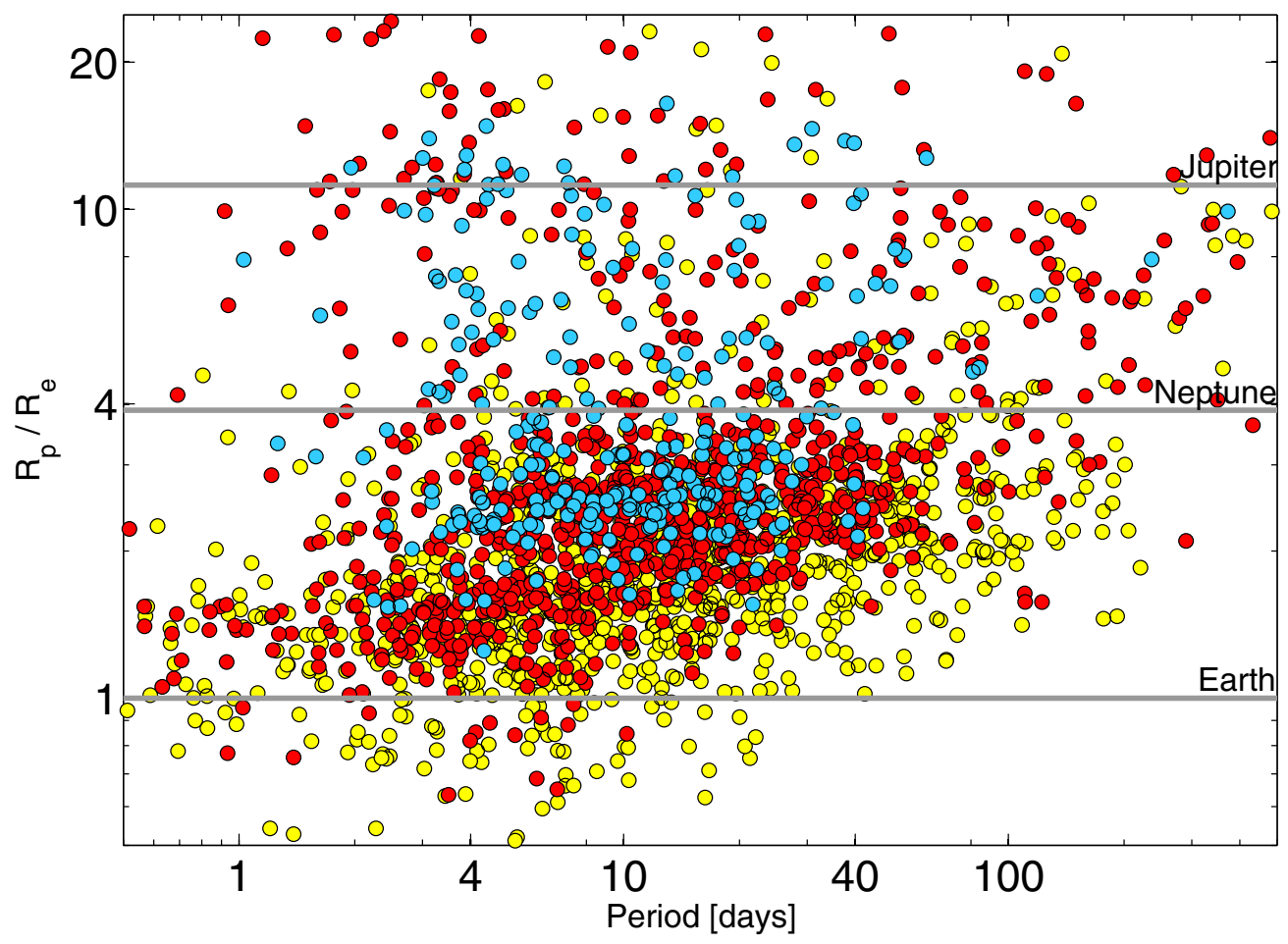

Figure 3. Radius vs. orbital period for each of the planet candidates in the B10 (Borucki et al. 2011a) catalog (blue points), the B11 (Borucki et al. $2011 \mathrm{~b})$ catalog (red points), and this contribution (yellow points). Horizontal lines marking the radius of Jupiter, Neptune, and Earth are included for reference.

\subsection{Period Aliasing}

Section 4 describes the vetting procedures and the many metrics used to eliminate false positives. One of the statistics is a measure of the significance of odd/even depth differences. Aside from identifying eclipsing binaries, the metric can also be used as a warning that a low- $\mathrm{S} / \mathrm{N}$ planetary period is a factor of two too low (as for KOI-730.03 and 191.04; Lissauer et al. 2011b). As an additional step outside the general procedures, we constructed a similar statistic for comparing the depths of every third or fourth transit. In this manner, the period of KOI1445.02 was revised to be a factor of three larger than initial estimates (54 days compared to 18 days as determined the pipeline). Table 4 contains the updated period value. In this exercise we identified a handful of other planet candidates with low $\mathrm{S} / \mathrm{N}$, primarily based on one or two transits each, ${ }^{33}$ with ephemerides predicting transits that are not detected. When only two transits are seen, it is possible that each is associated with a distinct planet candidate that only transits once, or, alternatively, that they are the primary and secondary eclipse of an eccentric, long-period, blended eclipsing binary. In some cases, there may be an alternative orbital period that phases the observed transits with a gap in the data take. More observations are required to determine a unique solution. These period aliases show that while the catalog is generally of high reliability, additional analyses on the light curves of low-S/N transits may generate revisions.

\section{DISTRIBUTIONS}

The metrics and procedures described above, as applied to the Q1-Q6 data, yield 1108 new planet candidates, representing

\footnotetext{
33 KOI-2224.02, 1858.02, and 2410.02 have ephemerides indicative of two transits in the light curve; KOI-1070.03 and 2410.01 have ephemerides dominated by one transit apiece.
}

a gain of $88 \%$ over the B11 catalog. Eight of the new candidates are single-transit events (as indicated by negative, integer period values in Tables 4 and 5). After removing the single-transit based candidates, the remaining candidates range in size from onethird the size of Earth to three times the size of Jupiter (transit depths of 20 parts per million to 20 parts per thousand) and equilibrium temperatures from $200 \mathrm{~K}$ to $3800 \mathrm{~K}$ (orbital periods of a half a day to nearly one year). Of the new candidates, 202 , 422,426 , and 40 are Earth-size $\left(R_{\mathrm{P}}<1.25 R_{\oplus}\right)$, super-Earthsize $\left(1.25 R_{\oplus} \leqslant R_{\mathrm{P}}<2 R_{\oplus}\right)$, Neptune-size $\left(2 R_{\oplus} \leqslant R_{\mathrm{P}}<\right.$ $\left.6 R_{\oplus}\right)$, and Jupiter-size $\left(6 R_{\oplus} \leqslant R_{\mathrm{P}}<15 R_{\oplus}\right)$, respectively. An additional 18 candidates are included in the catalog that are larger than $15 R_{\oplus}$, a small number of which are larger than three times the size of Jupiter and unlikely to be consistent with the planet interpretation. They are included here due to the uncertainties in the stellar radii (see Section 5.2). Section 7.3 describes the subsample of the new planet candidates that are in the HZ.

Figure 3 shows planet radius versus orbital period for the candidates in the B10 catalog (blue) and the B11 catalog (red) together with the new candidates reported here (yellow). The properties of all previously published KOIs have been updated as described in the Appendix. The range of the abscissa and ordinate in Figure 3 are truncated at 500 days and $25 R_{\oplus}$ (to more effectively display the population) thereby excluding 10 candidates, 2 of which display only a single transit. The points are layered (newest candidates underneath) so that the growing domain and range of each population is apparent. Not surprisingly, each successive catalog contains progressively smaller planet candidates at progressively longer orbital periods. The relative number of small-planet candidates is one of the striking features of the B11 catalog: over $73 \%$ of the candidates presented there are smaller than Neptune. This trend continues: in the current sample of new candidates, over $91 \%$ are smaller than Neptune. 

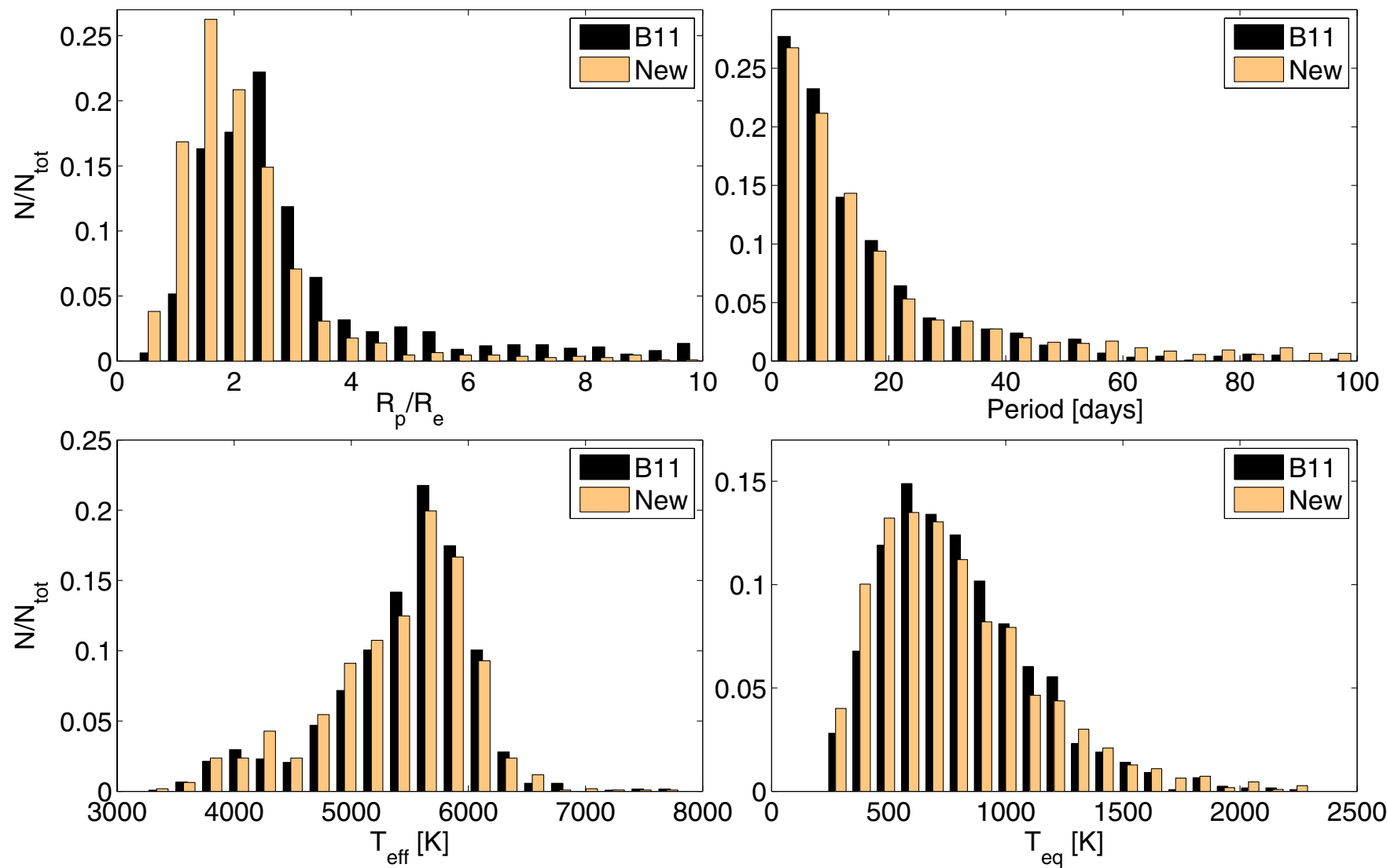

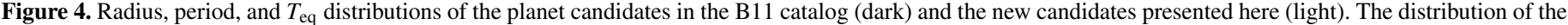
surface temperature of their host stars is also included. Counts are expressed as fractions of the total number of candidates in each of the two catalogs.

(A color version of this figure is available in the online journal.)

The relative gains in the number of candidates are displayed in Figure 4, which contains normalized distributions of planet radius, period, equilibrium temperature, and host star temperature for the B11 catalog and for the new candidates presented here. In terms of radius, the gains are predominantly for candidates smaller than $2 R_{\oplus}$. The new candidates contain a significantly smaller fraction of Neptune-size and Jupiter-size planets than the 2011 February catalog. We report a growth of $201 \%$ for candidates smaller than $2 R_{\oplus}$ compared to $53 \%$ for candidates larger than $2 R_{\oplus}$, and a growth of $124 \%$ for orbital period longer than 50 days compared to an $86 \%$ increase for periods shorter than 50 days. The gains in equilibrium temperature and host star properties are more uniform (approximately $88 \%$ for most bins). Section 7.1 compares these gains to what would be expected from increasing the baseline observation window from 13 months (Q1-Q5) to 16 months (Q1-Q6).

\section{DISCUSSION}

\subsection{Computed versus Observed Growth in Numbers of Candidates}

We compare the observed gain in the numbers of candidates to that expected from an increase in sensitivity afforded by three additional months of data. To do so, we develop a simplified model for the expected detection of transiting planets with Kepler data following Burke et al. (2006) whereby the detection probability is separated into two independent terms: probability for detection from a sensitivity $(\mathrm{S} / \mathrm{N})$ standpoint and probability for geometric alignment to transit.
The first term determines whether the data have sufficient photometric precision and number of observed transit events for detection above the $\sigma_{\text {thresh }}=7.1$ significance threshold adopted by the mission (Jenkins 2002). We estimate the total $\mathrm{S} / \mathrm{N}$ of a transit sequence of depth, $\Delta$, and orbital period, $P_{\text {orb }}$ as

$$
\mathrm{S} / \mathrm{N}=\frac{\Delta}{\sigma_{\mathrm{cdpp}}} \sqrt{N_{\mathrm{events}}},
$$

where $\sigma_{\mathrm{cdpp}}$ is the observed photometric noise for a given star on a timescale comparable to the transit duration. The duration is computed for a given stellar mass and assuming a circular orbit with period, $P_{\text {orb }}$, and an impact parameter $b=(\pi / 4)$ corresponding to the expectation value for an isotropic distribution of angular momentum vectors. The TPS pipeline algorithm measures $\sigma_{\mathrm{cdpp}}$ over a grid of transit durations from 1.5 to $15.0 \mathrm{hr}$ (Jenkins 2002; Christiansen et al. 2012), and we interpolate to determine the photometric noise at the estimated duration expected. The expected number of transit events is given by

$$
N_{\text {events }}=\eta \frac{T_{\mathrm{obs}}}{P_{\mathrm{orb}}},
$$

where $\eta=0.95$ is the duty cycle of the photometric time series and $T_{\text {obs }}$ is the duration of the observations.

The total $\mathrm{S} / \mathrm{N}$ of a transit event of a given depth, duration, and period associated with a star of specified characteristics is expressed as a detection probability. Since the detection statistic has unit variance (Jenkins 2002), the detection probability is the 


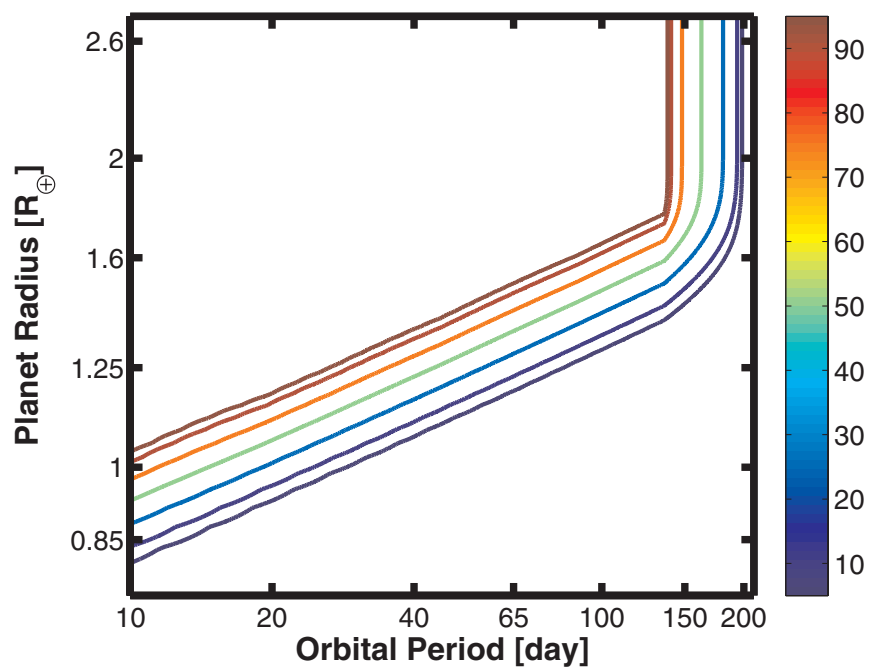

Figure 5. Detection probability (expressed as percent completeness) contours as a function of planet radius and orbital period for a $\mathrm{Kp}=12$ mag star with the properties listed in Table 6.

cumulative Gaussian distribution above $\sigma_{\text {thresh }}=7.1$ :

$$
P_{\mathrm{S} / \mathrm{N}}=\frac{1}{2}+\frac{1}{2} \operatorname{erf}\left[\frac{\left(\mathrm{S} / \mathrm{N}-\sigma_{\text {thresh }}\right)}{\sqrt{2.0}}\right] .
$$

We construct a grid of such probabilities as a function of transit depth and orbital period. Figure 5 shows a sample grid for a typical $\mathrm{Kp}=12$ magnitude star assuming five quarters of observations (407 days) and a 95\% duty cycle. The grid is displayed as contours of equal detection probability, or "percent completeness."

The TPS algorithm as implemented requires a minimum of three transit events for detection. To account for this in the detection probability we model the decrease in sensitivity toward longer $P_{\text {orb }}$ by determining the $P_{\text {orb }, 3}$ when $N_{\text {events }}=3$ and $P_{\text {orb,2 }}$ when $N_{\text {events }}=2$. The detection probability then linearly decreases from 1.0 to 0.0 probability over the range $P_{\text {orb }, 3} \leqslant P_{\text {orb }} \leqslant P_{\text {orb }, 2}$. This modeling of requiring three transits in the detection probability results in the apparent "upturn" of the detection probability contours at the longest orbital periods in Figure 5.

The product of the detection probability and the probability of geometric alignment yields the total detection probability. The geometric alignment probability is proportional to the ratio of the stellar radius and planet-star separation normalized to $0.46 \%$ for an Earth-Sun analog. Figure 6 displays (logarithmic) completeness contours for the same $\mathrm{Kp}=12$ star after including the geometric alignment probability. From left to right, the contours are $5.0 \%, 2.0 \%, 1.0 \%, 0.5 \%$, and $0.1 \%$, or $0.7,0.3,0.0$, -0.3 , and -1 , respectively, on the logarithmic scale. If nature produces planets that uniformly populate the radius/period plane with circular orbits, then the planets detected by Kepler will be distributed similarly to the contours shown.

Instead of computing a grid of total detection probabilities for each of the $>150,000$ stars in the parent sample, we devise a representative sample. Stars observed by Kepler, in the range $4.0<\log g<4.9$ and $R_{\star}<1.4$, are sorted into $\mathrm{Kp}=0.25 \mathrm{mag}$ bins, excluding those that do not have stellar classifications in the KIC. This results in 145,728 targets with $10.75<\mathrm{Kp}<17.75$. Table 6 lists the number of stars in each magnitude bin together with the median stellar radius for that bin. The associated $\log g$ and $T_{\text {eff }}$ are interpolated of Tables 15.7 and 15.8 in Cox

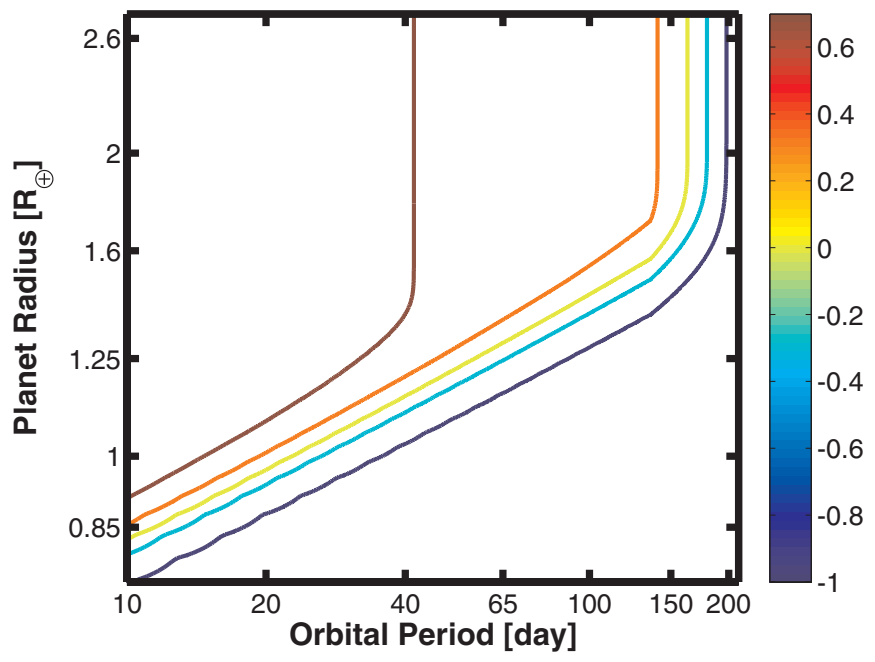

Figure 6. Probability contours displayed in Figure 5 are corrected for the geometric alignment probability (normalized to the geometric alignment probability of an Earth/Sun analog: 0.00465). These alignment-corrected probability contours are displayed here. The colors of the contours are mapped to the logarithm of the probability. These values, from left to right are $0.7,0.3,0.0,-0.3$, and -1 .

Table 6

The Parent Star Sample: Representative Properties and Star Counts

\begin{tabular}{lllcrr}
\hline \hline $\mathrm{Kp}$ & $R_{\star}$ & $\log g$ & $T_{\text {eff }}$ & Nstars & CDPP $^{\mathrm{a}}$ \\
\hline 11 & 1.144 & 4.3689 & 6055.0 & 315 & 23.3 \\
11.5 & 1.394 & 4.3440 & 6968.3 & 1282 & 27.4 \\
12 & 1.32 & 4.3462 & 6737.4 & 2327 & 31.1 \\
12.5 & 1.273 & 4.3481 & 6545.2 & 4177 & 36.9 \\
13 & 1.204 & 4.3547 & 6268.2 & 7272 & 44.5 \\
13.5 & 1.139 & 4.3705 & 6039.7 & 11892 & 55.2 \\
14 & 1.028 & 4.4303 & 5833.3 & 14961 & 69.0 \\
14.5 & 0.947 & 4.4744 & 5667.0 & 19340 & 92.0 \\
15 & 0.922 & 4.4816 & 5569.2 & 29831 & 123.3 \\
15.5 & 0.867 & 4.4856 & 5257.2 & 36818 & 168.9 \\
16 & 0.781 & 4.5081 & 4741.3 & 15900 & 221.2 \\
16.5 & 0.745 & 4.5249 & 4543.1 & 873 & 378.5 \\
17 & 0.738 & 4.5283 & 4505.5 & 603 & 532.6 \\
17.5 & 0.775 & 4.5107 & 4707.6 & 137 & 876.6 \\
\hline
\end{tabular}

Note. ${ }^{\text {a }} 30^{\text {th }}$ percentile of the $6 \mathrm{hr}$ CDPP values (in parts per million) of all stars in the relevant magnitude bin.

(2000). The photometric noise taken as the 30th percentile of the $6 \mathrm{hr}$ CDPP values ${ }^{34}$ of the targets in the magnitude bin. The stellar properties associated with the $\mathrm{Kp}=12$ mag bin are those used for the calculations that produced the results displayed in Figures 5 and 6. Though the majority of Kepler targets are G-type stars on or near the main sequence (Batalha et al. 2010b), it is evident from Table 6 that the median star type depends on magnitude. We assume that all stars in the magnitude bin can be represented by the properties tabulated. Though insufficient for computing Kepler's detection efficiency in an absolute sense, this assumption is a useful simplification for exploring the expected planet yield in a relative sense.

The total planet yield for a given magnitude bin is computed by taking the product of the alignment-corrected detection probability and the number of targets in that magnitude bin.

\footnotetext{
34 The distribution of CDPP values is very asymmetric with a long tail toward high CDPP values. The 30th percentile of the CDPP values was chosen as a proxy for the mode of the distribution to represent the typical photometric precision for a particular magnitude bin.
} 
Table 7

Observed versus Computed Gains in Planet Candidate Yield

\begin{tabular}{lccc}
\hline \hline & $1.25 R_{\oplus}<R_{\mathrm{P}}<2 R_{\oplus}$ & $2.5 R_{\oplus}<R_{\mathrm{P}}<6 R_{\oplus}$ & 10 days $<P<125$ days \\
\cline { 2 - 3 } & 5 days $<P<50$ days & 50 days $<P<150$ days & 1.00 \\
Computed $^{\mathrm{a}}$ & 1.12 & 1.35 & 1.6 \\
Observed $^{\mathrm{a}}$ & 2.8 & 7.2 & 1.6 \\
\hline
\end{tabular}

Notes. ${ }^{a}$ Gains are expressed as the ratio between the current total number of candidates (i.e., B 11 catalog plus the candidates presented here) and the number of candidates from the B11 catalog. Since the former is a product of the analysis of six quarters of data while the latter is a product of the analysis if five quarters of data, this is referred to in the text as $N_{\mathrm{Q} 6} / N_{\mathrm{Q} 5}$. The period and size range chosen for the rightmost column is where the B11 catalog is thought to be complete from a sensitivity standpoint (as indicated by a ratio near unity). Note that the observed ratio is significantly larger than unity even in this domain.

Summing these results for all magnitudes yields the total expected planet yield for a given period/radius combination assuming all stars have such a planet. We can then integrate over period and radius to obtain the planet yields in specific bins. These sums are weighted by the power-law distributions defined in Howard et al. (2012) to account for the fact that certain areas of the radius/period domain are more heavily populated with planets than others. Table 7 contains the expected versus observed gains in the numbers of candidates (expressed as a ratio, $N_{\mathrm{Q} 6} / N_{\mathrm{Q} 5}$, where $N_{\mathrm{Q} 5}$ is the number of candidates in the B11 catalog and $N_{\mathrm{Q} 6}$ is the total number of candidates, old plus new) for various radius/period bins. In every case, the observed gains are significantly larger than the predicted gains. Even in areas of the parameter space where we predict no gains (e.g., Neptune-size planets with periods shorter than 125 days where $N_{\mathrm{Q} 6} / N_{\mathrm{Q} 5}=1$ ), we observe appreciably more candidates.

This simplified exercise is meant to serve as a cautionary example for those performing statistical studies of planet occurrence rates as it demonstrates the incompleteness of the B11 catalog. ${ }^{35}$ The gains we observe cannot be explained by the longer observation window. More likely, the current batch of new planet candidates has benefitted from the growing sophistication of pipeline software, the most important of which is the multi-quarter functionality afforded by TPS for the first time in SOC 7.0. With multi-quarter functionality, data from different quarters are stitched together within pipeline modules before whitening and combing for transits. The analysis leading to the B11 catalog employed non-pipeline tools to do similar tasks via a modified BLS detection method. As such, the analysis did not make full use of the pipeline modules that carefully treat inter-quarter boundaries and perform the pre-search whitening of the light curves as described in Section 3. The absence of these modules has a significant impact on the detectability of shallow transits.

The SOC 7.0 pipeline also contains improvements to the module performing systematic-error correction (pre-search data correction, or PDC). Thermal transients occurring after monthly Ka-band downlinks and safe mode events introduce systematic errors in the light curves that are treated in the current pipeline. Left uncorrected, such transients have a negative impact of the detectability of transit events. Figure 5 of DRN 5 (KSCI-19045001) at MAST illustrates an example of a thermal transient after a safe mode event that is properly corrected by the new version of PDC. The BLS analysis applied to multi-quarter data in B11 circumvented this by masking out data points associated with thermal transients (12 events totaling 54.7 days).

\footnotetext{
35 This exercise is not, however, intended to produce accurate estimates of pipeline completeness for use in statistical studies. Readers are cautioned against overinterpreting the ratios presented in Table 7.
}

Collectively, these pipeline improvements lead to increased detection efficiency compared to previous efforts.

An important question is the degree to which the current catalog also suffers from incompleteness. Figure 7 shows the distribution of Q1-Q6 detection statistics (MES) for the cumulative list of planet candidates (new plus old). A powerlaw increase in the planet radius distribution toward smaller radii translates to a power-law increase the MES distribution (toward smaller MES values). The turnover at an MES of 9.7 suggests that incompleteness is likely to still be an issue. Planned pipeline upgrades already underway are expected to yield another significant improvement. For example, there are known limitations to PDC whereby astrophysical features that are distorted both in amplitude and frequency thereby precluding the detection of shallow planet transits. DRN 4 and 5 provide numerous examples, though Figure 9 of DRN 5 is especially relevant. Future pipeline improvements to mitigate these distortions are already under development.

There is a concerted effort to produce more uniform vetting procedures with each planet catalog release and to develop the tools that will allow for a quantitative assessment of catalog reliability, pipeline completeness, and planet occurrence rates. Such analyses are in progress.

\subsection{The New Multiples}

The first multiple transiting planet systems were identified in Kepler data within the first year of science operations (Borucki et al. 2011a; Steffen et al. 2010; Holman et al. 2010). One third of the 1235 candidates in the B11 catalog, or $17 \%$ of the 997 unique stars are host to multiple planet candidates. Evidence of dynamical interactions in the form of transit timing variations (TTVs) has been identified for approximately 100 candidates (Ford et al. 2012), and dynamical modeling of those variations has successfully produced mass determinations for five of the planets associated with Kepler-11 (Lissauer et al. 2011a). TTVs have proven useful for confirming and characterizing planets in multiple systems. Moreover, statistical analysis of multiples indicates that nearly all ( $\gtrsim 98 \%)$ are bona fide planets (Lissauer et al. 2012). That is, the false-positive rate for candidates transiting stars with multiple transiting planet candidates is significantly smaller than for single planet candidates. As such, the "multis" are a particularly reliable sample for future study.

As discussed in Section 3, all of the KOIs were inspected for evidence of additional transiting planet candidates. The search yielded 302 new candidates in multiple systems. Their properties are included in Tables 4 and 5. Combining the new candidates with the B11 catalog, we have 2338 candidates associated with 1797 unique stars. Three hundred sixty-nine of the 1797 stars host multiple candidates. Nine hundred ten of 


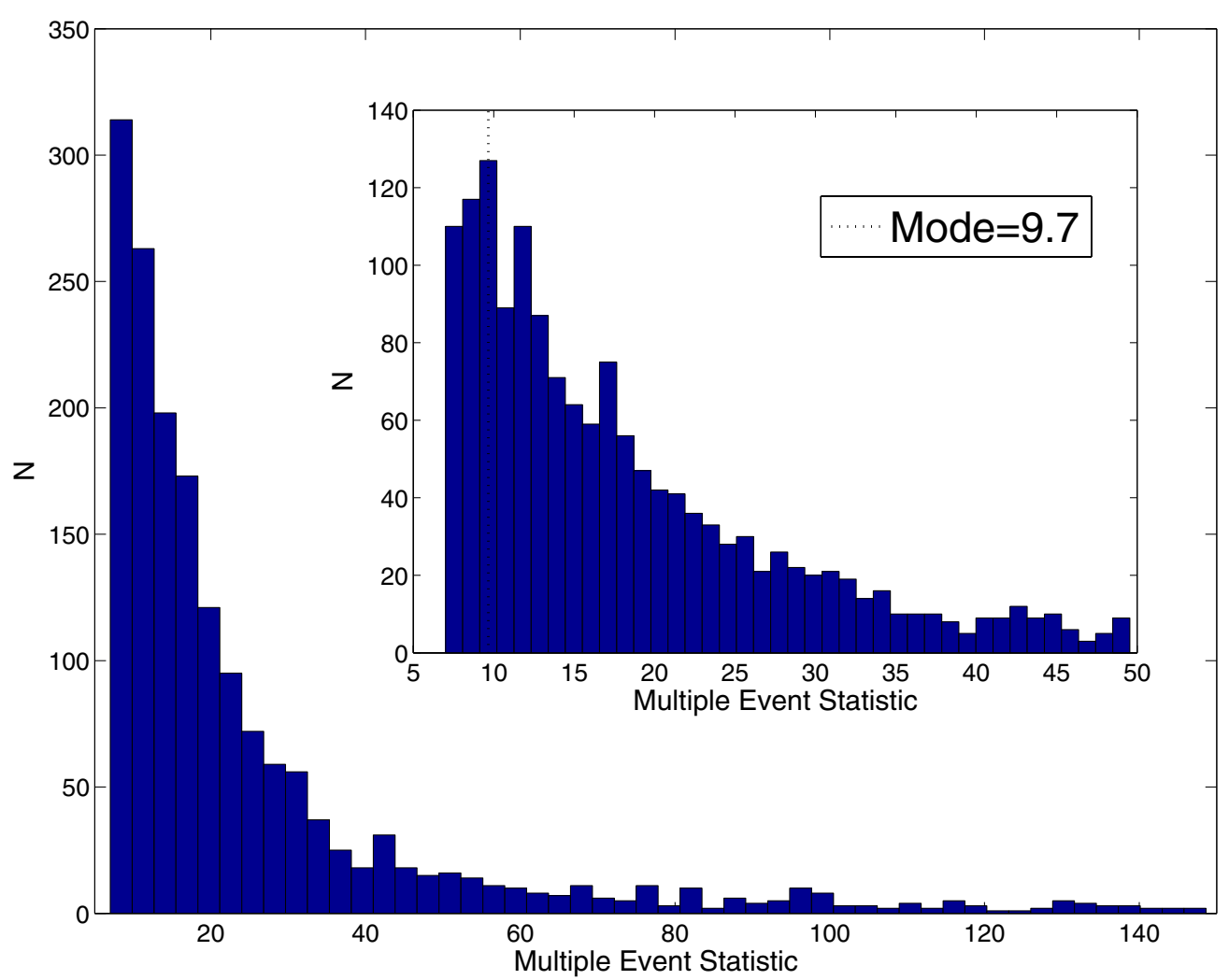

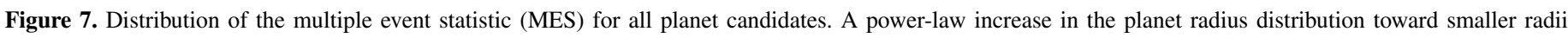

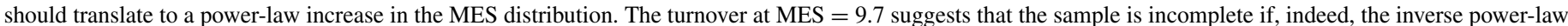
distribution of Howard et al. (2012) holds for the smallest planets.

(A color version of this figure is available in the online journal.)

the 2338 candidates are part of multiple systems. The fraction of KOI host stars with multiple candidates has risen from $17 \%$ to $20 \%$.

In Figure 8, we return to the cumulative list of candidates in the radius versus period plane, this time distinguishing candidates belonging to one (black), two (green), three (blue), four (yellow), five (cyan), and six (red) planet systems. The plot corroborates the observation made by Latham et al. (2011) in studying the multis in the B11 catalog: that there is a paucity of short-period giant planets in multiple systems. There is only one apparent exception to this-the green point near $R_{\mathrm{P}}=17 R_{\oplus}$, $P=3$ days (KOI-338.02). This is a new candidate associated with KOI-338, the first having been reported in the B11 catalog. Since that publication, spectroscopic observations have been acquired and subjected to spectral synthesis. The revised stellar parameters are $T_{\text {eff }}=4104 \mathrm{~K}$ and $\log g=1.87$ compared to $T_{\text {eff }}=4910 \mathrm{~K}$ and $\log g=4.18$ in the KIC. This results in a significant change in the estimated planet radius for KOI338.01 reported in B11. Re-evaluation of KOI-338.01 with the new stellar radius consistent with the lower surface gravity yields $R_{\mathrm{P}}=40 R_{\oplus}$. This transiting (eclipsing) object is too large to be planetary and is off the scale in Figure 8. The new candidate, KOI-338.02, is approximately Jupiter-size. A second spectroscopic observation from the same instrument (the TRES spectrograph on the Tillinghast Reflector at Whipple Observatory) was acquired, and yields a surface gravity that is more consistent with the KIC value. Additional observations are required to resolve the stellar properties. This target is discussed in more depth by Fabrycky et al. (2012).

We note that Figure 1 of Latham et al. (2011) contains other short-period giant planet candidates: the candidates associated with KOI-961. This high proper M dwarf from the proper motion catalog of Lepine \& Shara (2005) (LSPM J1928 + 4437) is unclassified in the KIC. In B11, the stellar radius was estimated by inferring an effective temperature from the $J-K$ color and assuming a main-sequence luminosity class. Improved stellar properties and light curve modeling of Muirhead et al. (2012b) result in a considerable decrease in the star and planet radii thereby removing these candidates from the upper left corner of Figure 8 and strengthening the case for an observed paucity of short-period giant planets in multiple systems.

Application of the statistical arguments presented by Lissauer et al. (2012) to the current population of multis implies that there are over 880 (98\% of 898 ) bona fide planets in this sample alone. A comprehensive study of the architecture of these multis is presented by Fabrycky et al. (2012).

\subsection{Candidates in the Habitable Zone}

Borucki et al. (2011b) identified 54 transiting planet candidates in the $\mathrm{HZ}$, defined by an equilibrium temperature, $T_{\mathrm{eq}}$ (see Section 5.3), between the freezing and boiling point of water $(273-373 \mathrm{~K})$ at standard pressure. As pointed out by Kasting (2011a), this definition fails to take into consideration the warming effect of an atmosphere, thereby rendering many of the candidates too hot for habitability even under the most liberal assumptions about their climatic conditions. As the roster of small planets at long orbital periods grows, so has the attention paid to the issue of habitability. Recent modeling efforts suggest that the equilibrium temperature at the inner edge of the HZ might be closer to $270 \mathrm{~K}$ (Selsis et al. 2007), corresponding to rapid water loss via $\mathrm{H}$ escape or a runaway greenhouse effect, depending on the surface water content. The outer edge depends 


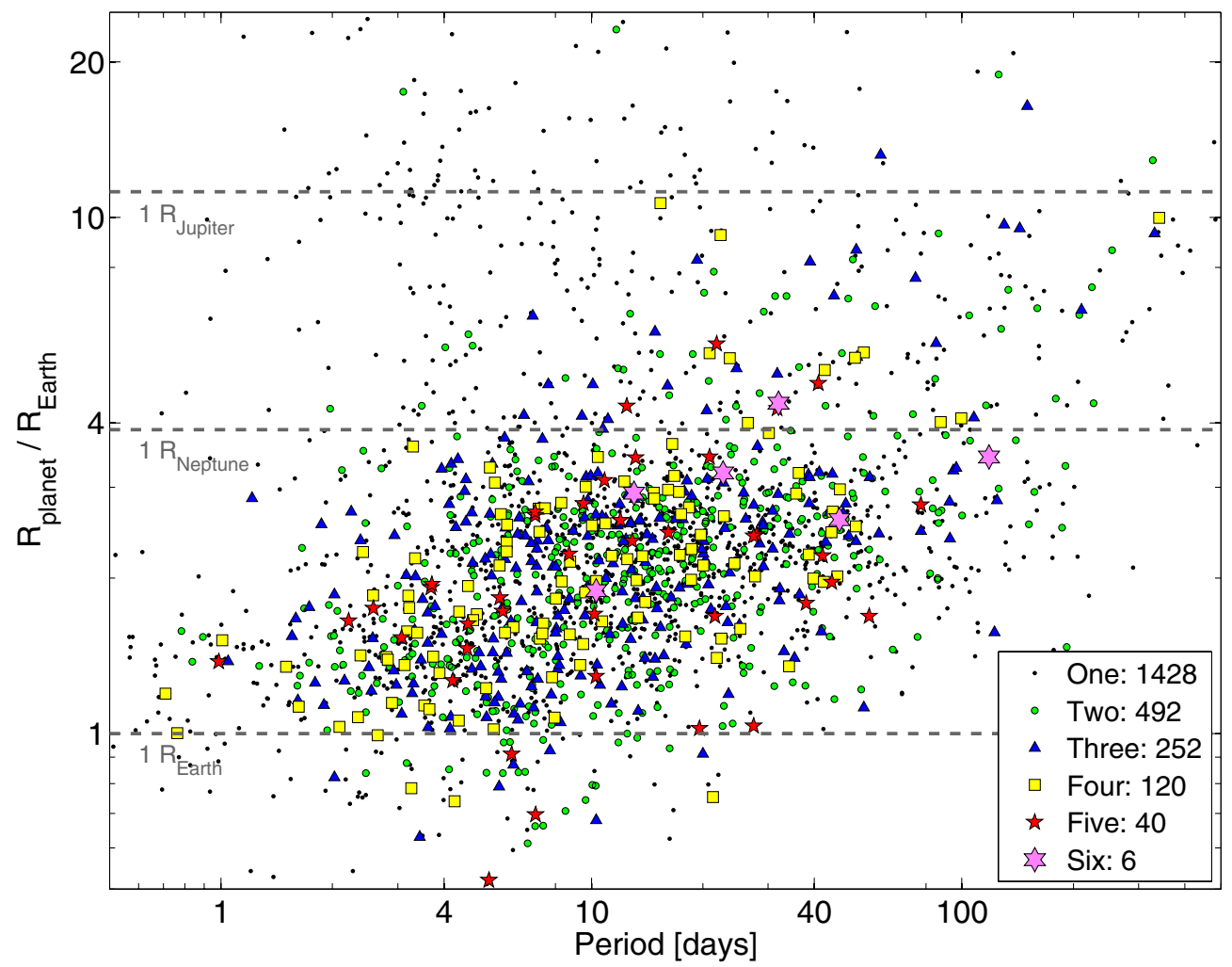

Figure 8. Planet radius vs. orbital period of the cumulative set of planet candidates as displayed in Figure 3. The points are colored to display members of one-, two-, three-, four-, five-, and six-planet candidate systems. We note the continued paucity of giant planets at short orbital periods in multiple planet systems. $20 \%$ of the stars cataloged have multiple planet candidates.

on the fractional cloud coverage and ranges from $175 \mathrm{~K}$ to $200 \mathrm{~K}$ (Kaltenegger \& Sasselov 2011). Others have proposed defining the $\mathrm{HZ}$ in terms of insolation (the amount of stellar flux incident on the planet surface) in order to remove the built-in assumptions (e.g., Bond albedo) required to compute the equilibrium temperature (Domagal-Goldman 2012).

The star and planet properties provided in Tables 3, 4, and 5 can be used to compute the insolation and/or equilibrium temperature under different assumptions for the purpose of assessing questions of habitability on a case-by-case basis. Here, we use equilibrium temperature as defined in Section 5.3 to examine the population of planet candidates likely to be in or near the HZ. Figure 9 shows planet radius versus equilibrium temperature for the entire sample of planet candidates. For reference, we include a vertical dashed line (middle) to mark the equilibrium temperature of the Earth computed under the same set of assumptions. With each new catalog (blue to red to yellow points), we see a clear trend toward Earth-size planets at Earth's equilibrium temperature (i.e., toward the bottom left hand corner of the diagram).

Figure 10 displays the same for the range $180 \mathrm{~K}<$ $T_{\text {eq }}<310 \mathrm{~K}$. The dotted vertical lines (far left and far right) mark the (generous) HZ boundaries (185-303 K) proposed by Kasting (2011b). The intermediate dashed vertical line marks the equilibrium temperature of the Earth under the same set of assumptions. There are 46 candidates in this temperature range (compared to 22 in the B11 catalog). Nine are super-Earth-size $\left(1.25 R_{\oplus} \leqslant R_{\mathrm{P}}<2 R_{\oplus}\right)$ and one is Earth-size $\left(R_{\mathrm{P}}<1.25 R_{\oplus}\right)$. Table 8 lists the properties for the 24 new candidates in this temperature range that are plotted in Figure 10. Note that candidates with only one transit event in the Q1-Q6 period are excluded from this list and Figures 9 and 10.
We have paid special attention to candidates that are in this temperature range and are also near Earth-size (see, for example, the discussion of KOI-326, KOI-364, and KOI-1026 in the Appendix). Figure 11 shows the relative flux time series of KOI-2124.01, the smallest viable candidate in this temperature range.

Table 8 contains two sets of stellar parameters. They are identical when spectroscopic values are available (as indicated by the flag, $f_{T_{\text {eff }}}$, in Table 3 ). They differ where KIC values were updated using a parameter search in the Yonsei-Yale stellar evolution models as described in Section 5.2. For the sample of stars listed in Table 8, the updates almost always lead to smaller stellar radii (and, hence, smaller and cooler planet candidates). Improved stellar characterization is required for a more reliable determination of the candidate location relative to the HZ.

\subsection{Citizen Science: Planet Hunters Discoveries}

PlanetHunters.org is a citizen science tool (Fischer et al. 2012), based on the Zooniverse platform (Lintott et al. 2008), that enables the search for transit events in the public Kepler data. The site serves up plots of Kepler light curves broken into 30 day segments, and, through a sequence of queries, leads the user through a high-level classification that sorts light curves by their qualitative properties, or appearance. Discerning eyes flag events that resemble transits, and the goal is to have every light curve examined by at least five independent users. Since its launch in 2010 December, over 10 million classifications have been made by over 100,000 users, underscoring the remarkable enthusiasm of the general public. The site affords one not only the opportunity to experience the scientific method but also the possibility of experiencing the gratification of discovery. With 


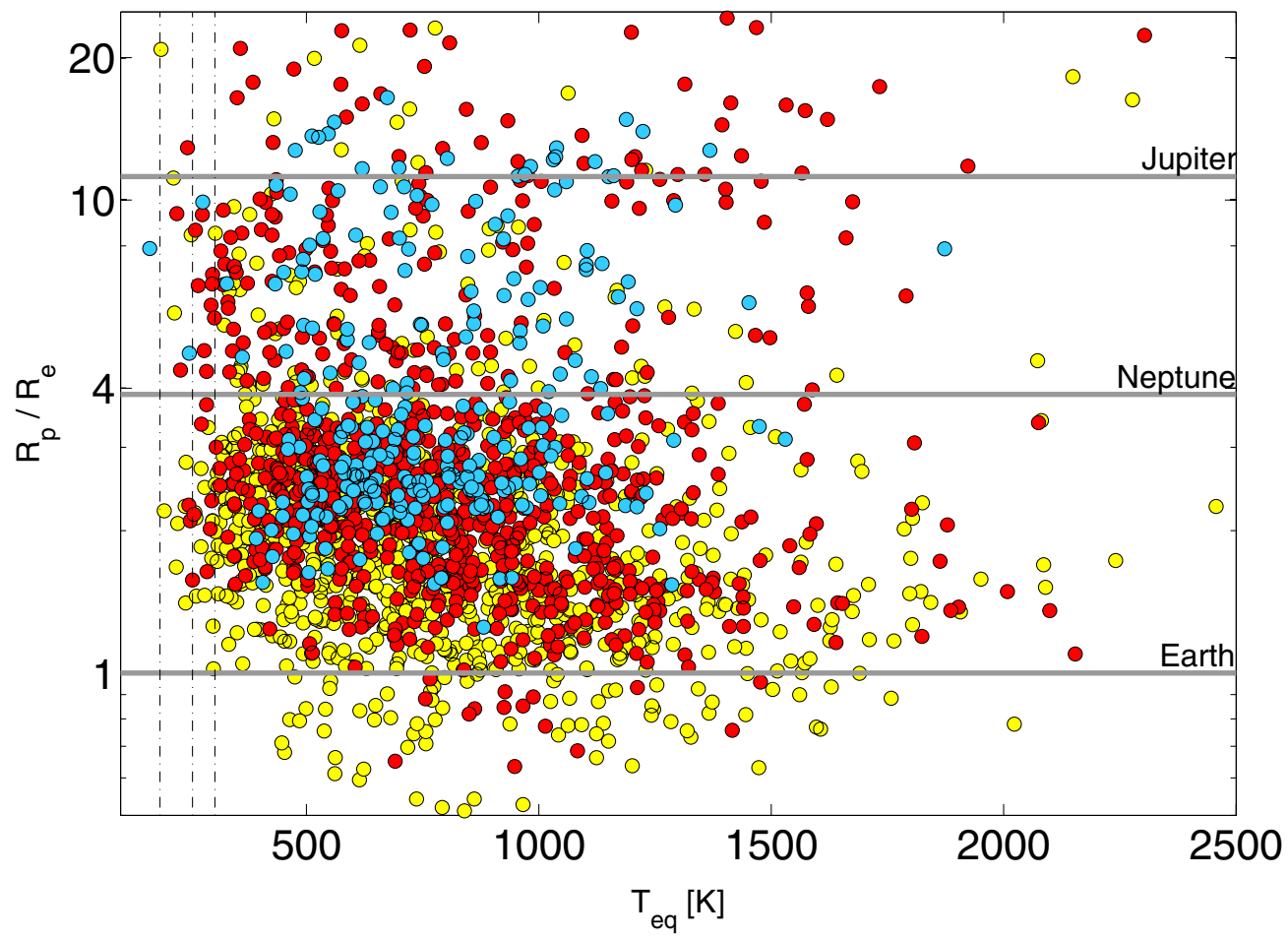

Figure 9. Radius vs. equilibrium temperature for each of the planet candidates in the B10 catalog (blue points), the B11 catalog (red points), and this contribution (yellow points). Horizontal lines marking the radius of Jupiter, Neptune, and Earth are included for reference. Also included for reference are vertical lines marking the inner and outer edges of the habitable zone as defined by Kaltenegger \& Sasselov (2011) as well as the equilibrium temperature for an Earth-Sun analog (middle line) under the same assumptions as those described in Section 7.3.

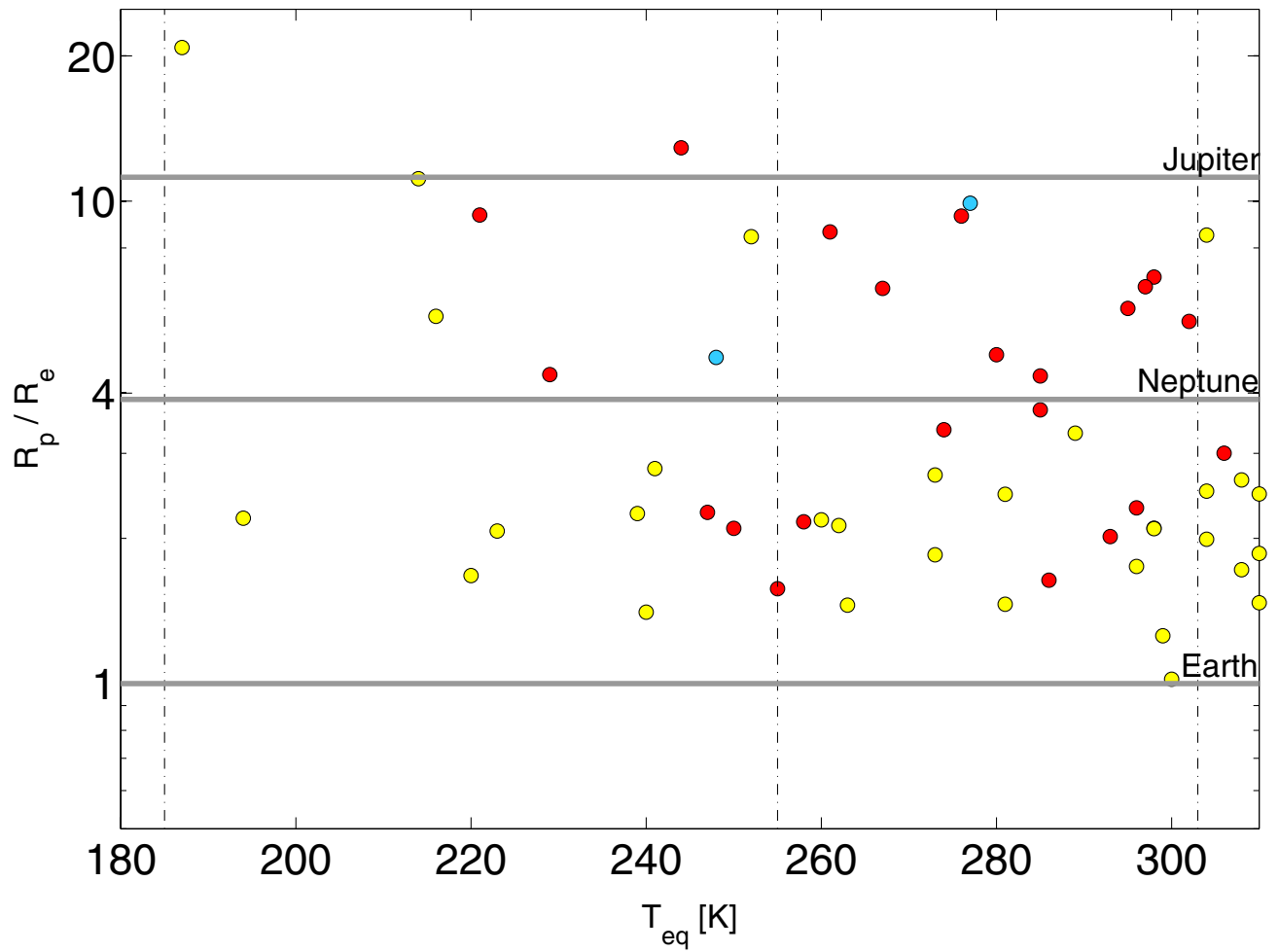

Figure 10. Same as Figure 9 for planet candidates in and near the habitable zone.

its power in numbers, the citizen science project is a welcome complement to the automated detection algorithms in Kepler's software pipeline.

The Planet Hunters science team combines the results from the multiple classifications for each 30 day light curve segment to identify potential planet candidates within the publicly released Kepler data. Transit-like events flagged by the public are assessed by the Planet Hunters science team. The Kepler project office then assists in vetting further for false positives. This process resulted in the identification of four potential planet 
Table 8

Planet Candidates with $185 \mathrm{~K}<T_{\mathrm{eq}}<303 \mathrm{~K}$

\begin{tabular}{|c|c|c|c|c|c|c|c|c|c|c|c|c|c|}
\hline KOI & $\begin{array}{l}\text { Period } \\
\text { (days) }\end{array}$ & $\begin{array}{c}R_{\mathrm{P}} \\
\left(R_{\oplus}\right)\end{array}$ & $\begin{array}{l}T_{\text {eq }} \\
(\mathrm{K})\end{array}$ & $O / E_{1}$ & Offset & MES & $\mathrm{S} / \mathrm{N}$ & $\begin{array}{c}T_{\text {eff }^{\mathrm{a}}} \\
(\mathrm{K})\end{array}$ & $\log g^{\mathrm{a}}$ & $\begin{array}{c}R_{\star}{ }^{\mathrm{a}} \\
\left(R_{\odot}\right)\end{array}$ & $\begin{array}{c}T_{\text {eff }} \text { b } \\
(\mathrm{K})\end{array}$ & $\log g^{\mathrm{b}}$ & $\begin{array}{c}R_{\star}{ }^{\mathrm{b}} \\
\left(R_{\odot}\right) \\
\end{array}$ \\
\hline 119.02 & 190.313 & 3.30 & 289 & 0.5 & 2.0 & -99 & 63.2 & 5380 & 4.44 & 1.0 & 5380 & 4.45 & 0.9 \\
\hline 438.02 & 52.662 & 2.10 & 298 & 0.6 & 1.0 & -99 & 26.7 & 4351 & 4.59 & 0.7 & 4351 & 4.71 & 0.6 \\
\hline 986.02 & 76.050 & 8.46 & 199 & 0.0 & 1.3 & -99 & 15.3 & 5250 & 6.85 & 4.5 & 5250 & 6.85 & 4.5 \\
\hline 1209.01 & 272.070 & 5.77 & 216 & 0.0 & 0.6 & -99 & 45.6 & 5316 & 4.79 & 0.6 & 5316 & 4.71 & 0.6 \\
\hline 1430.03 & 77.481 & 2.47 & 281 & 1.1 & 0.7 & 11.5 & 18.8 & 4502 & 4.60 & 0.7 & 4502 & 4.67 & 0.6 \\
\hline 1431.01 & 345.161 & 8.44 & 252 & 1.5 & 3.1 & -99 & 164.2 & 5649 & 4.46 & 1.0 & 5649 & 4.46 & 1.0 \\
\hline 1466.01 & 281.564 & 11.13 & 214 & 2.0 & 3.2 & -99 & 209.8 & 4768 & 4.45 & 0.9 & 4768 & 4.53 & 0.8 \\
\hline 1686.01 & 56.867 & 1.41 & 240 & 2.6 & 2.5 & 7.0 & 7.6 & 3665 & 4.47 & 0.7 & 3665 & 4.74 & 0.5 \\
\hline 1739.01 & 220.657 & 1.85 & 273 & 0.4 & 1.8 & 7.2 & 7.1 & 5677 & 4.63 & 0.8 & 5677 & 4.57 & 0.8 \\
\hline 1871.01 & 92.725 & 2.18 & 260 & 0.0 & -99 & 20.2 & 18.3 & 4449 & 4.65 & 0.7 & 4449 & 4.68 & 0.6 \\
\hline 1876.01 & 82.532 & 2.25 & 239 & 0.6 & 0.8 & 19.8 & 33.3 & 4230 & 4.39 & 0.9 & 4230 & 4.77 & 0.5 \\
\hline 1902.01 & 137.861 & 20.83 & 187 & 0.1 & 1.3 & 18.0 & 26.2 & 3818 & 4.50 & 0.7 & 3818 & 4.73 & 0.5 \\
\hline 1938.01 & 96.915 & 2.10 & 298 & 0.5 & 1.2 & 16.7 & 40.3 & 5071 & 4.66 & 0.7 & 5071 & 4.67 & 0.7 \\
\hline 2020.01 & 110.966 & 2.07 & 223 & 0.3 & 1.0 & 14.6 & 31.8 & 4350 & 4.47 & 0.8 & 4350 & 4.77 & 0.5 \\
\hline 2102.01 & 187.746 & 2.79 & 241 & 0.9 & 0.6 & 12.6 & 18.1 & 5100 & 4.50 & 0.9 & 5100 & 4.64 & 0.6 \\
\hline 2124.01 & 42.337 & 1.02 & 300 & 0.1 & 0.6 & 12.1 & 18.0 & 4103 & 4.51 & 0.7 & 4103 & 4.73 & 0.5 \\
\hline 2290.01 & 91.502 & 1.75 & 296 & 1.1 & 1.3 & 9.9 & 17.7 & 4969 & 4.89 & 0.5 & 4969 & 4.67 & 0.7 \\
\hline 2418.01 & 86.830 & 1.67 & 220 & 1.0 & 0.9 & 8.9 & 13.5 & 3863 & 4.26 & 1.0 & 3863 & 4.74 & 0.5 \\
\hline 2469.01 & 131.190 & 2.13 & 262 & 1.5 & 0.7 & 8.5 & 17.5 & 4727 & 4.42 & 1.0 & 4727 & 4.59 & 0.7 \\
\hline 2474.01 & 176.830 & 1.45 & 263 & 0.5 & 1.8 & 8.5 & 12.1 & 5284 & 4.60 & 0.8 & 5284 & 4.60 & 0.7 \\
\hline 2626.01 & 38.098 & 1.46 & 281 & 0.2 & 2.4 & 7.2 & 10.7 & 3735 & 4.51 & 0.6 & 3735 & 4.73 & 0.5 \\
\hline 2650.01 & 34.988 & 1.26 & 299 & 0.4 & 0.6 & 7.1 & 11.4 & 3900 & 4.50 & 0.7 & 3900 & 4.74 & 0.5 \\
\hline 2770.01 & 205.383 & 2.20 & 194 & 0.4 & -99 & 10.7 & 18.9 & 4352 & 4.64 & 0.6 & 4352 & 4.69 & 0.6 \\
\hline 2841.01 & 159.391 & 2.70 & 273 & 0.4 & -99 & 8.8 & 13.2 & 5213 & 4.64 & 0.8 & 5213 & 4.60 & 0.8 \\
\hline
\end{tabular}

Notes. Columns 1-8 are defined in Tables 4 and 5. Unavailable or invalid entries are assigned -99 .

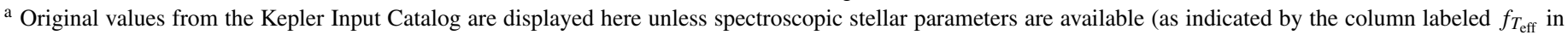
Table 3).

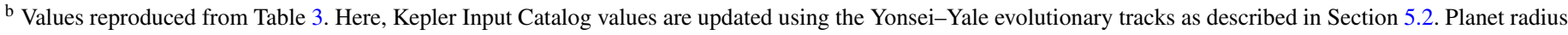
and equilibrium temperature are derived using these stellar properties.

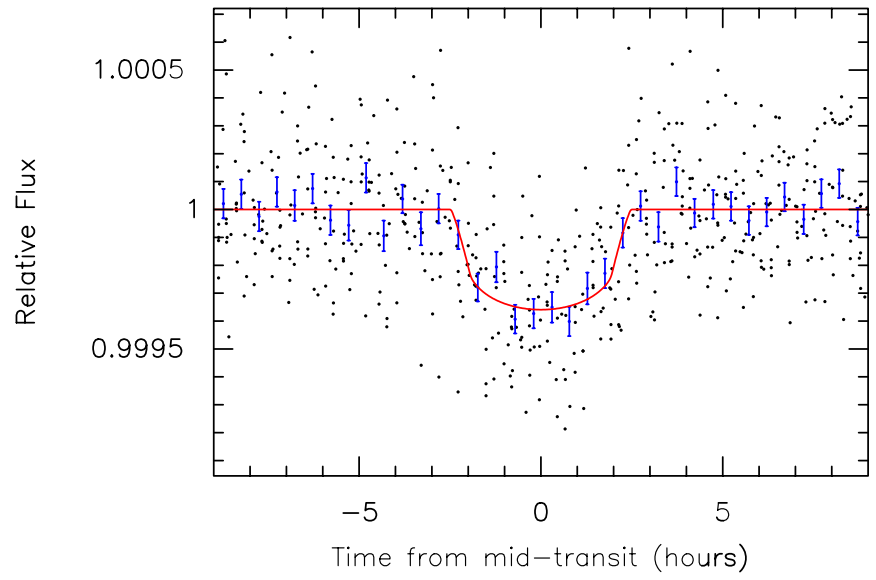

Figure 11. Phase-folded, relative flux time series of KOI-2124.01 light curve. KOI-2124.01 is the smallest viable planet candidate near the HZ.

(A color version of this figure is available in the online journal.)

candidates in the Q1 public data (Fischer et al. 2012), associated with stars KIC 10905746, KIC 8242434, KIC 6185331, and KIC 11820830, two of which (KIC 10905746 and KIC 6185331) were deemed viable candidates after inspection of vetting metrics. These four candidates were assigned KOI numbers (1725.01, 1726.01, 1727.01, and 1728.01, respectively), and blindly re-assessed together with the candidates identified here using Q1-Q6 data and the associated vetting metrics. All but 1728.01 survived as viable planet candidates. Their properties are listed in Tables 4 and 5. KOI-1728.01 was rejected due to the large radius of the companion $\left(>5 R_{\mathrm{J}}\right)$ and hints of ellipsoidal variations in the light curve indicative of a higher mass (i.e., stellar) companion. This target has been included in the Eclipsing Binary Catalog (Prša et al. 2011; Slawson et al. 2011).

More recently, inspection of the Q1 and Q2 public data by Planet Hunters led to another set of viable planet candidates associated with the stars KIC 4552729 and KIC 10005758 (Lintott et al. 2012). KIC 10005758 had already been assigned a KOI number due to the identification of a different transit event at a shorter period (KOI-1783.01). The new, longer period candidate identified by the Planet Hunters team has been assigned the KOI number 1783.02 while the candidate associated with KIC 4552729 has been assigned KOI number 2691.01. Detection statistics on KOI-1783.02 and KOI-2691.01 are not identified by the Q1-Q6 pipeline run due to (1) the long orbital period (KOI-1783.02) and (2) systematic noise sources that precluded identification of the correct orbital period (2691.01). The events are readily identified in a Q1-Q8 pipeline run that more recently became available, and all vetting statistics indicate that both are strong candidates. To maintain sample uniformity, we do not include these candidates in the tables presented here. They will, however, be included in future catalogs.

We note that nine of the candidates presented in Table 4 (1787.01, 1828.01, 1858.01, 1790.01, 1808.01, $1830.01,1613.01,1557.02,1930.04)$ were independently identified by Planet Hunters (KIC 5864975, KIC 11875734, KIC 8160953, KIC 6504954, KIC 7761918, KIC 3326377, KIC 6268648, KIC 5371776, and KIC 5511081, respectively) as described by Lintott et al. (2012). This further illustrates the potential for contributions from the citizen science community. 
The vetting statistics employed here and applied to the Planet Hunters candidates are quickly becoming an integrated part of a mature software pipeline. The Kepler team has worked to finetune these vetting metrics so that they are reliable and easily interpreted. The objective is to eventually make them publicly available so that users interested in identifying transits can also perform the vetting that is such an integral part of identifying viable planet candidates.

\section{SUMMARY}

We have analyzed pipeline results using Q1-Q6 data. Nearly 5000 TCEs were evaluated. Approximately 1500 were identified as objects of interest. Light curve modeling, DV pipeline results, and photo-center analysis yield metrics for vetting astrophysical false positives. The vetting process yielded nearly 700 new planet candidates. These as well as previous candidates were subjected to a modified BLS transit detection analysis after filtering the primary transit events. More than 300 additional candidates associated with multiple systems were identified in this way and subjected to the same vetting metrics.

We present 1108 new planet candidates and their properties (period, epoch, $R_{\mathrm{P}} / R_{\star}, d / R_{\star}$, and impact parameter) gleaned from light curve modeling. Planet radius and equilibrium temperature require knowledge of the stellar properties. Effective temperature and surface gravity from the KIC are used as input values to a Markov Chain Monte Carlo search for the best match Yonsei-Yale evolutionary model. The result is an estimate of the stellar radius. Forty-nine stars have a high-resolution spectrum obtained as part of the Kepler follow-up program. Analysis yields improved $T_{\text {eff }}, \log g$, and $R_{\star}$, the latter of which comes from the Yonsei-Yale models. Calculation of the planet radii yields 202, 422, 426, and 40 candidates that are Earth-size, super-Earth-size, Neptune-size, and Jupiter-size, respectively.

The distribution in both size and orbital period of the new candidates is qualitatively similar to that of the previously published candidates, although smaller planets are more prevalent. More than $91 \%$ of the new planet candidates are smaller than Neptune (compared to $73 \%$ for the B11 catalog). The largest relative gains are seen not only for the smaller planets but also for those at longer orbital periods. We report a growth of $201 \%$ for candidates smaller than $2 R_{\oplus}$, compared to $53 \%$ for candidates larger than $2 R_{\oplus}$, and a growth of $124 \%$ for orbital period longer than 50 days compared to an $86 \%$ increase for periods shorter than 50 days. The gains for the smaller planets cannot be explained by the modest increase in data collection. The observed gains exceed the computed gains even in regimes where one might expect previous catalogs to be complete (Table 7). This can be explained by improvements to the analysis pipeline, the most significant of which is the multi-quarter capability in the TPS and DV modules.

The fraction of stars with multiple transiting planet candidates has risen from $17 \%$ to $20 \%$. The cumulative list of 2338 viable planet candidates contains 1797 unique stars, 246 of which are two-planet systems, 84 of which are three-planet systems, 30 of which are four-planet systems, 8 of which are five-planet systems, and one of which is a six-planet system (Kepler-11; Lissauer et al. 2011a). A comparison of the single-planet systems with the multiple-planet systems shows a paucity of short-period $(\mathrm{P}<10$ days) giant planets in multiple systems as reported by Latham et al. (2011) and Steffen et al. (2012).

With each successive catalog, we see clear progress toward the Earth-size planets in the HZ (Figures 3 and 9). Twenty-five of the new candidates are located in the range
$185 \mathrm{~K}<T_{\text {eq }}<303 \mathrm{~K}$, and one, KOI-2124.01, is near Earthsize and at the hot end of this temperature range. The gains in the number of detections of planet candidates smaller than $2 R_{\oplus}$ will be a boon for studying occurrence rates. We proceed cautiously, however. Assuming that planet sizes are distributed according to an inverse power law (Howard et al. 2012; Youdin 2011), we would expect to see a similar distribution in the detection statistics of the candidates discovered to date. The turnover in the distribution just short of the detection threshold suggests that we will see further improvements in completeness as the pipeline continues to improve.

The authors express their sincere gratitude for the time and attention that both referees gave to this manuscript. In particular, we thank Dr. Scott Gaudi who offered a particularly thorough reading and valuable comments that significantly improved the manuscript. We were very fortunate to have benefitted from his expertise.

Funding for this Discovery mission is provided by NASA's Science Mission Directorate. This material is based on work supported by the National Aeronautics and Space Administration under grant NNX08AR04G issued through the Kepler Participating Scientist Program.

Facility: Kepler

\section{APPENDIX CUMULATIVE CATALOG OF PLANET CANDIDATES AND THEIR PROPERTIES}

We present a cumulative catalog of planet candidates and their properties. Candidates from the B11 catalog have been subjected to the same uniform modeling (as described in Section 5.1) using the same data set (Quarters 1-8) as the new candidates presented here. Moreover, the stellar parameters of their host stars have been updated in the same manner as described in Section 5.2. Over 300 of the host stars from previous catalogs have been observed spectroscopically as part of Kepler's ground-based follow-up program and subjected to the analyses that yield updated stellar properties used to derive the planet candidate properties. Updated ephemerides, light curve properties, modeled light curve parameters, derived planet characteristics, and stellar properties of all planet candidates are presented in Table 9. Updates to the catalog include (1) cases where period aliasing has been resolved, (2) cases where corrections for TTVs lead to improved light curve modeling, and (3) new ephemerides for some candidates which were listed in B11 as having only one observed transit but which have since presented additional transits.

We note that no comprehensive effort has yet been made to remove false positives from the B11 catalog based on followup observations and/or new data and vetting metrics. This will be done in a future contribution. There are, however, a small number of exceptions. For example, KOI-589.01 was reported in $\mathrm{B} 11$ as a $1.2 R_{\oplus}$ candidate with a 17.5 day orbital period and a total $\mathrm{S} / \mathrm{N}$ of $8.6 \sigma$. After adding new observations, the total $\mathrm{S} / \mathrm{N}$ of the signal has fallen below our detection threshold, to $3.7 \sigma$, suggesting that the event is a false alarm. Light curve modeling did not converge. The candidate has been omitted from Table 9. KOI-111.04 is a similar case. Reported in $\mathrm{B} 11$ as a $2.5 R_{\oplus}$ candidate in a 103.5 day orbit, this candidate now presents a total $\mathrm{S} / \mathrm{N}$ of just $4.2 \sigma$. It too, has been omitted.

Other exceptions are three candidates from B11 that are presumably small and in/near the HZ: KOI-326.01, KOI-364.01, 
Table 9

Description of Cumulative Planet Candidate Catalog

\begin{tabular}{|c|c|c|c|}
\hline Column & Format & Name & Description \\
\hline 1 & F7.2 & KOI & Kepler Object of Interest number \\
\hline 2 & I9 & $\mathrm{KIC}$ & Kepler Input Catalog identifier \\
\hline 3 & F7.3 & $\mathrm{Kp}$ & Kepler magnitude \\
\hline 4 & F10.5 & $T_{0}{ }^{\mathrm{a}}$ & Time of a transit center; BJD-2454900 \\
\hline 5 & F8.5 & $\sigma_{T 0}$ & Uncertainty in $T_{0}$ \\
\hline 6 & F12.7 & Period $^{\mathrm{a}}$ & Average interval between transits in days \\
\hline 7 & F10.7 & $\sigma_{P}$ & Uncertainty in period \\
\hline 8 & F6.2 & $R_{\mathrm{P}}^{\mathrm{b}}$ & Planetary radius in Earth radii $=6378 \mathrm{~km}$ \\
\hline 9 & F6.3 & $a^{\mathrm{c}}$ & Semimajor axis of orbit \\
\hline 10 & I5 & $T_{\mathrm{eq}}{ }^{\mathrm{d}}$ & Equilibrium temperature of planet \\
\hline 11 & F8.4 & $t_{\text {dur }}$ & Transit duration, first contact to last contact, in hours \\
\hline 12 & I6 & Depth & Transit depth at center of transit in parts per million \\
\hline 13 & F11.6 & $d / R_{\star}{ }^{\mathrm{e}}$ & Ratio of planet-star separation to stellar radius \\
\hline 14 & F11.6 & $\sigma_{d / R_{\star}}$ & Uncertainty in $d / R *$ \\
\hline 15 & F8.5 & $R_{\mathrm{P}} / R_{\star}$ & Ratio of planet radius to stellar radius \\
\hline 16 & F8.5 & $\sigma_{R_{\mathrm{P}} / R_{\star}}$ & Uncertainty in $r / R *$ \\
\hline 17 & F8.5 & $b^{\mathrm{f}}$ & Impact parameter of transit \\
\hline 18 & F8.5 & $\sigma_{b}$ & Uncertainty in $b$ \\
\hline 19 & F6.1 & $\mathrm{S} / \mathrm{N}^{\mathrm{g}}$ & Total S/N of all transits detected \\
\hline 20 & F5.2 & $\chi^{2}$ & Goodness of fit metric \\
\hline 21 & I5 & $T_{\text {eff }}$ & Stellar effective temperature \\
\hline 22 & F5.2 & $\log g$ & Log of stellar surface gravity \\
\hline 23 & F6.2 & $R_{\star} / R_{\odot}$ & Stellar radius \\
\hline 24 & I1 & $f_{T_{\mathrm{eff}}}^{\mathrm{h}}$ & Flag on $T_{\text {eff }}$ \\
\hline
\end{tabular}

Notes. No attempt has been made here to remove false positives from the table of Borucki et al. (2011b). Uniform vetting of these earlier KOIs is in progress.

${ }^{\text {a }}$ Based on a linear fit to all observed transits. For candidates with only one observed transit, the period is estimated from the duration and knowledge of the stellar radius; values are then rounded to the nearest integer and multiplied by -1 .

b Product of $r / R *$ and the stellar radius given in Table 1 .

c Based on Newton's generalization of Kepler's third law and the stellar mass computed from surface gravity and stellar radius and given in Table 3.

d See the main text for discussion.

e To first order, this parameter is equivalent to the ratio of the planet-star separation (at the time of transit) to the stellar radius. In the case of a zero-eccentricity orbit, it is equivalent to the reduced semimajor axis, $a / R_{\star}$.

${ }^{\mathrm{f}}$ Note that there is a strong covariance between $b$ and $d / R_{\star}$.

g $\mathrm{S} / \mathrm{N}$ of the phase-folded transit signal computed from modeling of Quarters 1-8 data.

${ }^{\mathrm{h}}$ Flag indicates source of $T_{\mathrm{eff}}, \log g$, and $R_{\star}$ as follows: (0) derived using KIC $J-K$ color and linear interpolation of luminosity class V stellar properties of Schmidt-Kaler (1982); (1) KIC $T_{\text {eff }}$ and $\log g$ are used as input values for a parameter search of Yonsei-Yale evolutionary models yielding updated $T_{\text {eff }}, \log g$, and $R_{\star} ;(2) T_{\text {eff }}, \log g$, and $R_{\star}$ are derived using SPC spectral synthesis and interpolation of the Yale-Yonsei evolutionary tracks; (3) $T_{\text {eff }}$, $\log g$, and $R_{\star}$ are derived using SME spectral synthesis and interpolation of the Yale-Yonsei evolutionary tracks.

(This table is available in its entirety in a machine-readable form in the online journal. A portion is shown here for guidance regarding its form and content.).

KOI-1026.01. KOI-326 (KIC 9880467) is unclassified in the KIC. The radius of the planet candidate reported in the B11 catalog assumed the host star is a main-sequence dwarf with an effective temperature defined by its $J-K$ color. However, subsequent spectroscopic follow-up observations indicate that the host star is more likely to be a giant. Preliminary estimates of the planet candidate radius are larger than $3 R_{\mathrm{J}}$. The KIC also lists an erroneous apparent magnitude in the Kepler bandpass for this star. The erroneous magnitude leads to a non-optimal photometric aperture precluding reliable photo-center analysis. This will be important since there is a brighter star (KIC 9880470) less than 5" away. More detailed analysis of KOI-364.01 and KOI-1026.01 suggests that the transit detection statistics are driven by systematics in the data. Pipeline improvements have led to a lower confidence in the planet interpretation. Consequently, these two candidates have been removed from the cumulative catalog. All three of these candidates will be monitored closely as more data become available.

The properties listed in Table 9 are derived from the (automated, bulk) light curve modeling described in Section 5.1. As of this writing, there are over 60 planet confirmations and characterizations in the literature based on Kepler transit detections. Such studies involve more data products and/or specialized analysis techniques that lead to improved planet properties that we do not attempt to catalog here. For example, KOI-1611.02 is the circumbinary planet published as Kepler-16ABb (Doyle et al. 2011). Since the uniform light curve modeling does not handle the case of circumbinary systems, most of the entries in Tables 4, 5, and 9 are assigned values of -99 indicating invalid parameters. This is an extreme example. However, all confirmed planets will have improved ephemerides and/or physical properties in the published literature. The properties of confirmed planets as well as the mapping between KIC, KOI, and Kepler 
identification numbers can be found at the NASA Exoplanet Archive. ${ }^{36}$

\section{REFERENCES}

Batalha, N. M., Borucki, W. J., Bryson, S. T., et al. 2011, ApJ, 729, 27 Batalha, N. M., Borucki, W. J., Koch, D. G., et al. 2010b, ApJL, 713, L109

Batalha, N. M., Rowe, J. F., Gilliland, R. L., et al. 2010a, ApJ, 713, 103

Borucki, W. J., Koch, D. G., Basri, G., et al. 2011a, ApJ, 728, 117

Borucki, W. J., Koch, D. G., Basri, G., et al. 2011b, ApJ, 736, 19

Borucki, W. J., Koch, D. G., Batalha, N., et al. 2012, ApJ, 745, 120

Brown, T. M., Latham, D. W., Everett, M. E., \& Esquerdo, G. A. 2011, AJ, 142,112

Bryson, S., Tenenbaum, P., Jenkins, J. M., et al. 2010, ApJ, 713, 97

Buchhave, L. A., Latham, D. W., Johansen, A., et al. 2012, Natur, 486, 375

Burke, C. J., Gaudi, S., DePoy, D. L., \& Pogge, R. W. 2006, AJ, 132, 210

Chaplin, W. J., Appourchaux, T., Elsworth, Y., et al. 2010, ApJL, 713, L169

Christiansen, J. L., Jenkins, J. M., Barclay, T. S., et al. 2012, PASP, submitted (arXiv:1208.0595)

Cox, A. N. (ed.) 2000, Allen's Astrophysical Quantities (4th ed.; New York: AIP)

Demarque, P., Woo, J.-H., Kim, Y.-C., \& Yi, S. K. 2004, ApJS, 155, 667

Derekas, A., Kiss, L. L., Borkovits, T., et al. 2011, Sci, 332, 216

De Ridder, J., Barban, C., Baudin, F., et al. 2009, Natur, 459, 398

Domagal-Goldman, S. 2012, ApJ, submitted

Doyle, L., Carter, J. A., Fabrycky, D. C., et al. 2011, Sci, 333, 1602

Fabrycky, D., Lissauer, J. J., Ragozzine, D., et al. 2012, ApJ, submitted (arXiv:1202.6328)

Fischer, D. A., Schwamb, M. E., Schawinski, K., et al. 2012, MNRAS, 419, 2900

Ford, E., Ragozzine, D., Rowe, J. F., et al. 2012, ApJ, 756, 185

Fressin, F., Torres, G., Rowe, J. F., et al. 2012, Natur, 482, 195

Gilliland, R. L., Brown, T. M., Christensen-Dalsgaard, J., et al. 2010, PASP, 122,131

Holman, M. J., Fabrycky, D. C., Ragozzine, D., et al. 2010, Sci, 330, 51

Howard, A. W., Marcy, G. W., Bryson, S. T., et al. 2012, ApJS, 201, 15

Jenkins, J. M. 2002, ApJ, 575, 493

Jenkins, J. M., Borucki, W. J., Koch, D. G., et al. 2010a, ApJ, 724, 1108

Jenkins, J. M., Chandrasekaran, H., McCauliff, S. D., et al. 2010b, Proc. SPIE, 7740, 77400D
Kaltenegger, L., \& Sasselov, D. 2011, ApJ, 736, 25

Kasting, J. F. 2011a, AsBio, 11, 363

Kasting, J. F. 2011b, Joint Meeting of the Exoplanet and Cosmic Origins Program Analysis Groups (ExoPAG and COPAG), 2011 April 26, Baltimore, MD, http://exep.jpl.nasa.gov/exopag/exopagCopagJointMeeting/

Kovács, G., Zucker, S., \& Mazeh, T. 2002, A\&A, 391, 369

Latham, D. W., Rowe, J. F., Quinn, S. N., et al. 2011, ApJL, 732, L24

Lepine, S., \& Shara, M. M. 2005, AJ, 129, 1483

Lintott, C., Schawinski, K., Slosar, A., et al. 2008, MNRAS, 389, 1179

Lintott, C., Schwamb, M. E., Barclay, T., et al. 2012, AJ, submitted (arXiv:1202.6007)

Lissauer, J. J., Fabrycky, D. C., Ford, E. B., et al. 2011a, Natur, 470, 53

Lissauer, J. J., Marcy, G. W., Rowe, J. F., et al. 2012, ApJ, 750, 112

Lissauer, J. J., Ragozzine, D., Fabrycky, D. C., et al. 2011b, ApJS, 197, 8

Mandel, K., \& Agol, E. 2002, ApJ, 580, 171

Mann, A. W., Gaidos, E., Lépine, S., \& Hilton, E. J. 2012, ApJ, 753, 90

Muirhead, P. S., Hamren, K., Schlawin, E., et al. 2012a, ApJL, 750, L37

Muirhead, P. S., Johnson, J. A., Apps, K., et al. 2012b, ApJ, 747, 144

Pinsonneault, M. H., An, D., Molenda-Żakowicz, J., et al. 2012, ApJS, 199, 30

Press, W. H., Teukolsky, S. A., Vetterling, W. T., \& Flannery, B. P. 1992, Numerical Recipes in Fortran 77 (2nd ed.; Cambridge: Cambridge Univ. Press), 678

Prša, A., Batalha, N., Slawson, R. W., et al. 2011, AJ, 141, 83

Quintana, E. V., Jenkins, J. M., Clarke, B. D., et al. 2010, Proc. SPIE, 7740, $77401 \mathrm{X}$

Schmidt-Kaler, T. 1982, in Landolt-Börnstein New Series, Group 6, Vol. 2b, Stars and Star Clusters, ed. K. Schaifers \& H.-H. Voigt (Berlin: Springer), 1

Selsis, F., Kasting, J. F., Levrard, B., et al. 2007, A\&A, 476, 1373

Slawson, R. W., Prša, A., Welsh, W. F., et al. 2011, AJ, 142, 160

Steffen, J. H., Batalha, N. M., Borucki, W. J., et al. 2010, ApJ, 725, 1226

Steffen, J. H., Ragozzine, D., Fabrycky, D. C., et al. 2012, PNAS, 109, 7982

Tenenbaum, P., Christiansen, J. L., Jenkins, J. M., et al. 2012, ApJS, 199, 24

Twicken, J. D., Chandrasekaran, H., Jenkins, J. M., et al. 2010b, Proc. SPIE, 7740,62

Twicken, J. D., Clarke, B. D., Bryson, S. T., et al. 2010a, Proc. SPIE, 7740,69

Valenti, J. A., \& Fischer, D. A. 2005, ApJS, 159, 141

Valenti, J. A., \& Piskunov, N. 1996, A\&AS, 118, 595

Wu, H., Twicken, J. D., Tenenbaum, P., et al. 2010, Proc. SPIE, 7740, 42

Youdin, A. N. 2011, ApJ, 742, 38

36 http://exoplanetarchive.ipac.caltech.edu 